\title{
Genetic and chemical validation of Plasmodium falciparum aminopeptidase PfA-M17 as a drug target in the hemoglobin digestion pathway
}

\author{
Rebecca C.S. Edgar ${ }^{1,2}$, Ghizal Siddiqui ${ }^{5}$, Kathryn Hjerrild', Tess R. Malcolm³, Natalie B. Vinh", \\ Chaille T. Webb ${ }^{3,5}$, Christopher A. MacRaild ${ }^{6}$, Natalie A. Counihan ${ }^{1,2}$, Darren J. Creek ${ }^{6}$, Peter J \\ Scammells ${ }^{4}$, Sheena McGowan ${ }^{3,5}$, Tania F. de Koning-Ward ${ }^{1,2^{*}}$ \\ ${ }^{1}$ School of Medicine, Deakin University, Geelong, 3216, Australia; \\ ${ }^{2}$ The Institute for Mental and Physical Health and Clinical Translation, Deakin University, \\ Geelong, 3216, Australia; \\ ${ }^{3}$ Biomedicine Discovery Institute and Department of Microbiology, Monash University, Clayton, \\ 3052, Australia; \\ ${ }^{4}$ Medicinal Chemistry, Monash Institute of Pharmaceutical Sciences, Monash University Parkville, \\ 3052, Australia; \\ ${ }^{5}$ Centre to Impact AMR, Monash University, Melbourne, 3800, Australia; \\ ${ }^{6}$ Drug Delivery, Disposition and Dynamics, Monash Institute of Pharmaceutical Sciences, Monash \\ University, Parkville, 3052, Australia \\ ${ }^{*}$ Correspondence to Tania F de Koning-Ward.
}

Email: taniad@deakin.edu.au

Keywords: malaria, aminopeptidase, hemoglobin digestion, conditional knockdown, drug development 


\begin{abstract}
Plasmodium falciparum, a causative agent of malaria, continues to remain a global health threat since these parasites have developed increasing resistance to all anti-malaria drugs used throughout the world. Accordingly, drugs with novel modes of action are desperately required to combat malaria. P. falciparum parasites infect human red blood cells where they digest the hosts main protein constituent, hemoglobin. Leucine aminopeptidase PfA-M17 is one of several aminopeptidases that have been implicated in the last step of this digestive pathway. Here we utilize both reverse genetics and a compound specifically designed to inhibit the activity of PfAM17 to show that PfA-M17 is essential for $P$. falciparum survival as it provides parasites with free amino acids for growth, many of which are highly likely to originate from hemoglobin. We further show that our inhibitor is on-target for PfA-M17 and has the ability to kill parasites at nanomolar concentrations. Thus, in contrast to other hemoglobin-degrading proteases that have overlapping redundant functions, we validate PfA-M17 as a potential novel drug target.
\end{abstract}

\title{
Introduction
}

Malaria is an infectious disease caused by protozoan parasites belonging to the Plasmodium genus, of which Plasmodium falciparum is the deadliest to humans. In 2019, there were more than 400,000 deaths attributed to malaria infections, the majority of these occurring throughout subSaharan Africa and South-East Asia (1). The current front-line therapeutic artemisinin and its derivatives, as well as partner drugs, are under threat as resistance to these drugs is spreading. Artemisinin resistance is now widespread throughout Asia, and has recently spread to Papua New Guinea, while resistance in malaria endemic regions of Africa emerged last year (2-4). The growing spread of resistance highlights the need to identify new therapeutic targets and compounds with novel modes of action (5).

The intra-erythrocytic cycle of $P$. falciparum is responsible for the clinical manifestations of disease and is the target of most antimalarials. Parasite survival during the erythrocytic stage is dependent 
upon the digestion of host hemoglobin to provide amino acids essential for parasite growth, with the exception of isoleucine, which is absent from human hemoglobin and, therefore, has to be taken up from the extracellular environment (6). Digestion of hemoglobin also creates space in the erythrocyte to accommodate the growing parasite, as well as providing a mechanism to regulate the osmotic pressure of the host cell (7-10). Hemoglobin digestion begins during the early ring stage of growth, but formation of hemozoin crystals, a detoxified version of the digestive by-product heme, can only be visualized by microscopy in the more developed trophozoite stage in a specialized acidic compartment termed the digestive vacuole (DV; (11). The DV contains an array of proteases responsible for the release of peptides from hemoglobin, including plasmepsins, falcipains, falcilysin and aminopeptidases (12); it is the latter which are speculated to perform the final step of amino acid release from these peptides (13).

As aminopeptidases are implicated in the final step of hemoglobin digestion, they are promising novel therapeutic targets. The $P$. falciparum genome encodes two neutral metalloaminopeptidases: the M1 alanyl aminopeptidase (PfA-M1) and M17 leucyl aminopeptidase (PfAM17). The broad-spectrum aminopeptidase inhibitor bestatin has been shown to kill $P$. falciparum, with parasites overexpressing PfA-M17 displaying resistance to this drug, suggesting PfA-M17 is its target in vivo $(14,15)$. Harbut et al. (2011) also showed that treatment of $P$. falciparum with bestatin reduced hemoglobin digestion and decreased isoleucine uptake. Specific inhibition of PfAM17 using an activity-based probe based on the bestatin scaffold resulted in ring-stage arrest and parasite death, whilst an equivalent probe designed to specifically inhibit PfA-M1 resulted in DV swelling and stalling of parasite growth much later at the trophozoite stage. This led the authors to conclude that PfA-M17 may be playing a role outside of, or in addition to, hemoglobin digestion (16). Several series of inhibitors designed to inhibit both PfA-M17 and PfA-M1 have also been developed and these suppress a range of Plasmodium species in vivo and in vitro (17-19). Drinkwater et al. (2016), for example, developed a series of dual inhibitors that killed sensitive and multi drug resistant parasites in the nanomolar range, validating PfA-M1 and PfA-M17 as potential novel therapeutic targets. 
PfA-M17 is a $68 \mathrm{kDa}$ cytoplasmic enzyme that forms a homo-hexamer in its active form, with optimal function at neutral $\mathrm{pH}$, similar to the $\mathrm{pH}$ of the parasite cytoplasm (20-22). Mathew et al. (2021) recently confirmed the cytoplasmic localization of PfA-M17, which supports the proposition that hemoglobin-derived peptides are exported from the DV into the parasite cytoplasm, either through the chloroquine-resistance transporter or by other unidentified mechanisms (23). Once in the cytoplasm, it is believed that hemoglobin-derived peptides are digested by PfA-M17, however, it is also possible that PfA-M17 plays an additional role in the catabolic turnover of peptides from other origins. PfA-M17 almost exclusively cleaves leucine and tryptophan in vitro, with leucine being one of the most abundant amino acids in adult hemoglobin $(24,25)$. Functionally, leucine has been shown to be an important substrate of the isoleucine transporter at the parasite membrane (26). As isoleucine is the only amino acid that is absent from hemoglobin and is sourced from the host serum, leucine generated by the parasite may be important for the uptake of isoleucine across the parasite membrane $(6,10)$. While repeated attempts to knockout PfA-M17 have failed $(20,27)$, suggesting it is essential for intra-erythrocytic growth, its ortholog could be disrupted in both Plasmodium berghei, a rodent malaria species, and in the closely related apicomplexan parasite, Toxoplasma gondii $(28,29)$.

In order to functionally characterize PfA-M17 and determine its contribution to $P$. falciparum survival, we created a conditional knockdown parasite line that enabled PfA-M17 expression to be regulated via a riboswitch (30). This revealed that parasites depleted of PfA-M17 experience a growth delay and fail to expand in culture, subsequently leading to parasite death. We have also designed, synthesized, and characterized a novel and specific small molecule inhibitor to PfA-M17, compound 3, and parasites treated with this inhibitor demonstrate a similar growth phenotype to PfA-M17 knockdown parasites. Finally, metabolomic analysis of knockdown and 3-treated parasites revealed that many peptides accumulating after the depletion of PfA-M17 are likely to originate from hemoglobin, indicating that PfA-M17 plays a role in the final stages of its digestion. 


\section{Results}

\section{Epitope tagging and incorporation of a $g / m S$ ribozyme into the PfA-m17 locus}

To tease out the function of PfA-M17 and determine its essentiality, we sought to use reverse genetics to deplete its expression using a conditional riboswitch system. Accordingly, the PfA-m17 locus was targeted by transfecting $P$. falciparum 3D7 with a pM17-HAglmS construct (Fig. 1A). Transfectants underwent three rounds of drug cycling with WR99210 before a pure population of integrated parasites was obtained by limiting dilution. Diagnostic PCR confirmed that these parasites were positive for pM17-HAglmS integration (Fig. 1B). Western blot analysis of whole parasite lysate confirmed expression of HA tagged PfA-M17, running slightly lower than the predicted $72 \mathrm{kDa}$ size (Fig. 1C). Immunofluorescence analysis (IFA) further confirmed HA expression, showing that PfA-M17 was excluded from both the food vacuole and the parasite nucleus (Fig. 2A). Sequential solubilization assays performed on mixed-stage parasite lysates showed that PfA-M17 was released into the soluble fraction and was absent from membraneassociated or integral membrane fractions, supporting its cytosolic localization (Fig. 2B). Western blot analysis of protein lysate harvested from Pf3D7 wild type parasites every 6 hours and probed with rabbit anti-M17 showed continuous expression throughout the asexual blood stages, with peak expression around $30 \mathrm{hpi}$ as previously reported. (Fig. 2C; See Fig. S1 for M17 antibody characterization).

\section{Knockdown of PfA-M17 expression results in altered parasite growth and a cycle fold- decrease}

The synthetic ribozyme incorporated into the 3'UTR of PfA-M17-HAglmS parasites allows the knockdown of protein expression at the transcriptional level with addition of glucosamine (GlcN), allowing characterization of protein function and assessment of the proteins contribution to parasite growth. Ring stage parasites at 0-4 hpi in cycle 1 were treated with $2.5 \mathrm{mM} \mathrm{GlcN}$ or left untreated. Parasites were harvested at trophozoite stage in cycle 1 and cycle 2 and significant protein knockdown was determined by Western blotting. This revealed that knockdown was efficient, with $84 \%$ and $92 \%$ knockdown in cycle 1 and 2, respectively (Fig. 3A). Parasite growth was determined 
by Giemsa-stained smears and compared to untreated parasites (Fig. 3B). Whilst there was no delay in parasite growth in cycle 1, a significant delay in parasite growth was observed the cycle following knockdown (C2) (Fig. 3B, 3C). This growth delay was already evident by early trophozoites stage and parasites reaching schizogony showed significant morphological changes. Few parasites went on to commence cycle 3 , as evidenced by the significantly different parasitemias at $100 \mathrm{~h}$ post-treatment (Fig. 3D). Measurement of parasite survival after 10 days in culture was also assessed using a Sybr Green I assay, which revealed knockdown with GlcN was significantly detrimental to parasite growth (Fig. 3E). None of these growth defects were evident in Pf3D7 parasites that had been treated with the same concentration of $\mathrm{GlcN}$, confirming that the effect was due to the loss of PfA-M17 (Fig. S2). Overall, this demonstrates that loss of PfA-M17 has a detrimental effect on parasite growth and indicates that this aminopeptidase is essential for survival of $P$. falciparum.

\section{Knockdown of PfA-M17 results in multiple digestive vacuoles forming during trophozoite stage but does not alter the quantity of hemozoin}

On analysis of PfA-M17-HAglmS parasites the cycle following knockdown, it was noted that some parasites at early trophozoite stage contained multiple digestive vacuoles (DV). To determine the significance of this finding, PfA-M17-HAglmS Giemsa stained parasites, alongside Pf3D7 parasites, were scored for their number of digestive vacuoles, indicated by hemozoin under light microscopy, the cycle after GlcN addition; only singly infected RBCs were counted, and the rest excluded (Fig 4A). PfA-M17-HAglmS knockdown parasites had significantly more DV per parasite compared to untreated parasites as determined by a non-parametric Dunn's post-hoc test (Fig. 4A). The addition of GlcN to Pf3D7 parasites did not affect DV numbers, indicating that this phenotype was not as a result of treatment with GlcN but specific to the loss of PfA-M17.

We next determined if the loss of PfA-M17 and increase in number of DV resulted in increased hemozoin formation, and thus hemoglobin digestion. PfA-M17-HAglmS and Pf3D7 parasites were harvested at developmentally similar early trophozoite stages the cycle following GlcN addition and the amount of hemozoin was measured. Between all groups there was no statistical difference after 
the addition of GlcN, and while there appeared to be an upward trend of the amount of hemozoin in GICN treated PfA-M17-HAgImS parasites compared to untreated, this did not reach significance (Fig. 4B). Thus, while the loss of PfA-M17 increases the number of DV per parasite, it does not increase the quantity of free heme, representative of haemoglobin digestion and hemozoin production, suggesting that there is not an upstream compensatory effect on this digestive pathway.

\section{A specific inhibitor of PfA-M17 kills parasites within a nanomolar range}

Since knockdown of PfA-M17 resulted in parasite death, we next sought to develop and characterize a specific inhibitor to target PfA-M17 and to validate it as on-target using our knockdown parasite line. PfA-M17 uses a metal dependent mechanism to hydrolyze the scissile peptide bond of peptide substrates, removing single amino acids from the $\mathrm{N}$-terminal of short peptides. Our parallel program of inhibitor design and synthesis has identified a number of hydroxamic acid-based compounds that inhibit PfA-M17(19, 31).

A potent and selective PfA-M17 inhibitor, 3 was synthesized from the aryl bromide 1, that we have reported previously(31). The synthesis first involved the attachment of the 4-hydroxymethylphenyl moiety via a Suzuki coupling reaction to afford 2 (Fig. 5A). The ester of 2 was subsequent converted to the corresponding hydroxamic acid via treatment with hydroxylamine hydrochloride under basic conditions (Fig. 5A). The inhibitory activity toward purified, recombinant PfA-M17 shows 3 to be a potent inhibitor $\left(K_{i}=18 \pm 3 n M\right)$ with excellent selectivity over PfA-M1 $\left(K_{i}=4424 \pm 501 \mathrm{nM}\right.$, Fig.S3), a different aminopeptidase that often shows cross-reactivity with PfA-M17 substrates and inhibitors $(19,31)$. Analysis of the $2.5 \AA$ X-ray crystal structure of PfA-M17 bound to 3 showed that the position and orientation of the compound was similar to our previous inhibitors with the hydroxamic acid core coordinating to the zinc ions in the active site (Fig. 5B, Fig. S3C). The 4hydroxymethylphenyl packed with the hydrophobic residues Leu487, Gly489, Leu492 Met396, Phe583 and Ala577 in the S1 pocket and interestingly, the hydroxyl group of $\mathbf{3}$ appears to interact with the sulfur atom of Met392 (Fig. 5B). The pivalamide occupied the expected position within the S1' pocket. Further detail and analysis of structure-activity relationships of $\mathbf{3}$ can be found in Supplementary text. 
With the inhibitor in hand, its effectiveness on parasites was next tested. The $\mathrm{EC}_{50}$ of 3 on Pf3D7 was determined to be $326 \mathrm{nM}(145-581 \mathrm{Cl})$ using a standard 72-hour killing assay, whereby parasite survival was determined over a range of compound concentrations (Fig. 5C). To determine at what stage of the erythrocytic cycle $\mathbf{3}$ impacts on parasite growth, parasite cultures were treated with either $10 \mathrm{xEC}_{50} 3$ or artesunate $\left(\mathrm{EC}_{50} 4.0 \mathrm{nM}, 0.5-6.5 \mathrm{Cl}\right)$ for 24 or 48 hours, commencing at either the ring or trophozoite stage, and following compound washout, cultures were incubated for a further 48 hours (Fig. 5D). This revealed that 3 was less effective at killing parasites with only a 24-hr treatment period compared to the 48-hr treatment, irrespective of whether the compound was administered at ring or trophozoite stage. Treatment for only 24 hours was significantly more effective when added at the trophozoite stage compared to ring stage addition. The most effective killing for 3 was observed when trophozoite stage parasites were treated for 48 hours. These findings were consistent with the observation that expression of PfA-M17 peaks within the rapid trophozoite growth stage, with much lower expression during the ring stage. Moreover, it also corresponds with the growth delay observed at trophozoite stage after knockdown of PfA-M17 protein expression. Analysis of Giemsa-stained P\&3D7 parasites treated with $5 x$ or $10 x$ the $\mathrm{EC}_{50}$ showed that both treatments resulted in a growth delay commencing around early trophozoite stage when compared to the vehicle control DMSO-treated parasites and parasites had either not entered schizogony (10x EC 50 treatment) or only just completed schizogony ( $5 \times \mathrm{EC}_{50}$ treatment) 64 hours later (Fig. 5E). Further analysis determined that Pf3D7 parasites, upon developing into trophozoites, also developed multiple digestive vacuoles (DV), as seen with specific knockdown of PfA-M17, when treated with either $5 \mathrm{x}$ or $10 \mathrm{x}$ the $\mathrm{EC}_{50}$ of 3 (Fig $5 \mathrm{~F}$ ).

\section{Analysis of parasites depleted of PfA-M17 alongside treatment with the specific PfA-M17 inhibitor}

To determine the specificity of $\mathbf{3}$ for PfA-M17 and whether it exhibits off-target effects, we next examined the sensitivity of parasites depleted of PfA-M17 to 3. For these experiments, PfA-M17HAglmS parasites depleted of PfA-M17 using $2.5 \mathrm{mM} \mathrm{GlcN}$ were treated with $5 \mathrm{x}$ or $10 \mathrm{x}$ the $\mathrm{EC}_{50}$ of $\mathbf{3}$ the cycle following knockdown at the point where the growth delay becomes apparent in GlcN 
treated parasites (Fig. 3B) and the percentage growth compared to PfA-M17 expressing parasites (ie. not exposed to GlcN treatment) treated with DMSO or $5 x$ the $\mathrm{EC}_{50}$. Analysis of growth using Sybr Green I assay showed that there was no significant difference in growth curves between parasites depleted of PfA-M17 and those additionally treated with 3 (Fig. 6A). The reduction in growth at $94 \mathrm{hpi}$ of all treated lines is consistent with the observation that parasites fail to reinvade and commence a subsequent cycle, unlike parasites treated with DMSO alone (Fig. 6A). Moreover, analysis of Giemsa-stained PfA-M17-HAglmS parasites showed that the addition of 5x or 10x the $\mathrm{EC}_{50}$ of 3 in the cycle following depletion of PfA-M17 showed a comparative phenotype to PfA-M17 knockdown parasites treated with DMSO (Fig. 6B). These results are in keeping with 3 being ontarget and that the effect of this compound on parasites is likely to be through inhibition of PfA-M17.

\section{Metabolomic analysis of parasites depleted of PfA-M17 or treated with 3 indicates PfA-M17 plays a role in the degradation of hemoglobin-derived peptides.}

To further confirm the specificity of $\mathbf{3}$ and to determine why knockdown of PfA-M17 has a drastic effect on parasite growth, we compared the metabolomic profiles of PfA-M17 parasites and Pf3D7 parasites grown in the presence and absence of GlcN and Pf3D7 parasites treated with 10x the $\mathrm{EC}_{50}$ of 3 for 1 hour. Principle component analysis and heatmap analysis of relative abundances of putative metabolites dysregulated following PfA-M17 knockdown and parasites treated with 3 showed that the most prominent metabolomic signature shared between them was a series of peptides that were increased (Fig. 7). This was then confirmed by two independent metabolomics experiments of PfA-M17 depletion (Fig. S4), and treatment with 3 (Fig. S5). Targeted analysis of the common set of peptides identified in all three experiments demonstrated that the majority (80 of 149 common peptides) of them are significantly ( $p$-value $<0.05)$ dysregulated in abundance in parasites depleted of PfA-M17 and following treatment with 3 (Fig. S6). Further detailed analysis of the significantly dysregulated 80 peptides demonstrated that PfA-M17 depletion or treatment with 3 increases the abundance (fold change $>1.5$ ) of these peptides, with the exception of three peptides (Lys/Gly, Glu/Glu/Glu/Lys/Trp, and Asp/Phe/lle/Tyr/Tyr; indicated by black bar) that were only enriched following treatment with 3 (Fig. 8A). These 80 peptides were then analyzed to 
determine whether they could be derived from hemoglobin $(\mathrm{Hb}) \alpha, \beta$, or $\delta$, and $\sim 82 \%$ of these peptides could be mapped to one of the $\mathrm{Hb}$ species (yellow dots; Fig. 8B). Thus, metabolomics analysis of parasites depleted of PfA-M17 or treated with a M17 specific inhibitor suggests that PfAM17 is highly likely to catalyze a terminal stage of $\mathrm{Hb}$ digestion.

\section{Parasites grown in amino acid free medium containing only isoleucine become sensitized to the PfA-M17 inhibitor 3}

Since a function of hemoglobin digestion is to provide free amino acids to the parasite, we next determined if removal of exogenous amino acids, with the exception of isoleucine, from the culture medium would sensitize parasites to 3 . A standard 72-hour killing assay was used to determine the $E_{50}$ of the compound on Pf3D7 parasites cultured concurrently in normal medium containing all amino acids, or amino acid free medium containing only isoleucine. This showed that parasites became significantly more sensitive to $\mathbf{3}$ in amino acid free media, indicating that its target PfAM17 is responsible for supplying parasites with amino acids essential for survival (Fig. 9). Comparatively, the loss of exogenous amino acids did not significantly impact the $\mathrm{EC}_{50}$ of Artemisinin over $72 \mathrm{~h}$ as has previously been shown(16).

\section{Discussion}

For many years PfA-M17 has been implicated in the final stage of hemoglobin digestion without any definitive confirmation. Here, using a well-established conditional system to knockdown expression of PfA-M17, we demonstrate that a specific loss of this protein corresponds to delayed growth and eventual stalling of parasite development upon transition into trophozoite stage. The resulting phenotype is fatal, with parasites unable to propagate and advance through further cycles, consistent with previous conclusions that PfA-M17 is likely to be an essential protein on the basis that Pfa-m17 is refractory to gene knockout $(20,27)$. This validates PfA-M17 as a potential novel drug target. 
Unlike previous studies on PfA-M17, which have utilized external activity-based probes or compounds, the conditional knockdown strategy negates any off-target effects that may have convoluted the dissection of its function. Using the approaches herein, we were additionally able to show that 3, specifically designed to target PfA-M17, was on-target, and parasites treated with this compound displayed a similar phenotype and metabolic profile to parasites in which PfA-M17 expression had been conditionally depleted. Analysis of the significantly disrupted metabolites after PfA-M17 knockdown or treatment with 3 revealed an increase in abundance of peptides, many of which are likely to be derived from hemoglobin. This suggests that the failure to generate a sufficient pool of some amino acids from hemoglobin through a reduction in aminopeptidase activity may be the cause of parasite death. That $P$. falciparum parasites were significantly more sensitized to 3 when cultured in the absence of exogenous amino acids also suggests that the main function of PfA-M17 is to provide amino acids for parasite growth.

Harbut et al. (2011) previously showed that $P$. falciparum cultured in the presence of an activitybased probe designed to inhibit PfA-M17 resulted in parasite death at the early ring stage of the parasite lifecycle, suggesting that the role of PfA-M17 in hemoglobin digestion may be subservient to an additional but essential role it plays earlier in the lifecycle (16). The authors hypothesized this additional role is in the turnover of dipeptides originating from the proteosome, as proteasome inhibitors display ring-stage killing (33). While we cannot rule out the latter since some of the peptides that were upregulated upon PfA-M17 knockdown did not map to hemoglobin, this appears not to be the overriding function of PfA-M17 and instead the different stages at which parasites stall in the presence of the activity-based probe are likely to stem from off-target effects.

Teasing out the function of PfA-M17 has been complicated by the fact that another aminopeptidase, PfA-M1, has also been implicated in the final stages of hemoglobin digestion. Initial studies localized PfA-M1 to the digestive vacuole, leading to speculation that the two aminopeptidases play a similar role in different parasite compartments (20). However, it is now clear that PfA-M1 is also cytoplasmic (22). Interestingly, inhibition of PfA-M1 using an affinity-based probe resulted in parasites stalling at the trophozoite stage (16), not dissimilar to the phenotypes observed herein 
upon knockdown of PfA-M17 or treatment with 3. However, treatment with this PfA-M1 affinitybased probe also resulted in swelling of the digestive vacuole, a phenotype that we did not observe with PfA-M17 knockdown. This phenotype has previously been seen when enzymes within the digestive vacuole implicated at the earlier stages of hemoglobin digestion have been targeted by compounds (16, 34). Given that PfA-M17 and PfA-M1 are both cytoplasmic proteins, this has begged the question whether there is redundancy between the two, as has previously been shown with other enzymes involved in hemoglobin digestion such as falcipains and plasmepsins (10). Our results, however, indicate that loss of PfA-M17 cannot be compensated for, and as pfa-m1 has also been previously shown to be impervious to gene knockout, this would suggest that both aminopeptidases are required for parasite survival $(20,27)$. A thorough analysis of the P1 substrate specificities of recombinant PfA-M17 and PfA-M1 gives some indication as to why this may be. PfAM1 is a monomeric clan MA alanyl aminopeptidase with broad substrate specificity (25). In contrast, PfA-M17 is a clan MF leucine aminopeptidase with a far narrower specificity for the hydrophobic amino acids, with a strong preference for a P1 leucine and tryptophan residue(25). The inhibitor 3 takes advantage of this specificity with a biphenyl ring system bound in the $\mathrm{S} 1$ pocket satisfying the hydrophobic preferences of PfA-M17, but with an addition of a hydroxymethyl group to allow the inhibitor to reach beyond the S1 and pick up an interaction with the S-atom of Met392 (Fig $\mathrm{S} 3 \mathrm{C})$. Taken together, the inhibitor specificity combined with the P1 substrate preference for leucine suggests PfA-M17 may be a dedicated aminopeptidase for processing leucine, with leucine being the most abundant amino acid in human haemoglobin (24).

Since our results indicate that PfA-M17 digests peptides derived from hemoglobin, it is not surprising that this enzyme is not essential in the closely related apicomplexan parasite, Toxoplasma gondii, which does not infect red bloods cells and therefore is not exposed to hemoglobin (29). However, the homologous leucine aminopeptidase is also not essential in the rodent malaria species, $P$. berghei (28). Analysis of the PbA-M17 recombinant enzyme identified that it had a similar substrate specificity to that of $P f A-M 17$, however, there were notable differences in substrate specificity of the Pb-M1 vs PfA-M1 enzymes, suggesting a difference in aminopeptidase substrates between the two species (35). Knockout of PbA-M17 did, however, 
result in a significant delay in growth, although the significance of this cannot be inferred given the small sample number of two mice (28). Notably, isoleucine is present in mouse hemoglobin but absent in human hemoglobin. In $P$. falciparum-infected red blood cells, the influx of extracellular isoleucine can be mediated by leucine, which serves as a substrate of the isoleucine transporter at the parasite membrane $(25,26)$. Since PfA-M17 has been demonstrated to have considerable specificity for leucine (25) and $P$. berghei can source isoleucine from hemoglobin, this may explain why PfA-M17 is essential for $P$. falciparum survival whilst $P$. berghei can survive without PbA-M17.

A novel finding observed after specific depletion of PfA-M17 was the presence of multiple digestive vacuoles per parasite, a phenotype not previously seen with inhibition of aminopeptidases in $P$. falciparum. Whether this is because parasites attempt to endocytose more red blood cell cytoplasm to try and salvage their free amino acids pool is unknown and will require further investigation in order to elucidate the mechanism of this finding. We also saw this phenotype in parasites treated with 3, further suggesting that our compound is on target for PfA-M17. We did not see a significant difference in the amount of hemozoin produced between parasites expressing or depleted of PfAM17, and a similar finding was observed between $P$. berghei wildtype and pba-m17 knockout parasites. This indicates that depletion or loss of leucine aminopeptidases may not have an impact further upstream in the hemoglobin digestion pathway resulting in altered hemozoin production.

In conclusion, we have demonstrated that PfA-M17 plays an essential role in the survival of $P$. falciparum and is likely playing a major role in the release of amino acids originating from hemoglobin, confirming PfA-M17 as a promising target for future anti-malarial drugs. Further analysis into additional metabolites found not to map to a hemoglobin sequence will provide insight into their origin and clues as to additional function(s) of PfA-M17. Encouragingly, we have found that $\mathbf{3}$ is able to kill parasites in a nanomolar range. An X-ray crystal structure of recombinant PfAM17 shows binding to this compound and addition of $\mathbf{3}$ to parasites after knockdown of PfA-M17 did not alter the parasite growth or morphology, suggesting the inhibitor is specific for PfA-M17. 3 also appears to be highly selective for PfA-M17 over PfA-M1 and this compound, now validated, provides scope for the further analysis into the function of PfA-M17, negating problems that arise 
with the death phenotype of the knockdown and providing a rationale for further development of inhibitors against PfA-M17.

\section{Materials and Methods}

Chemistry. Methyl 2-(4'-(hydroxymethyl)-[1,1'-biphenyl]-4-yl)-2-pivalamidoacetate (2). To a mixture of methyl 2-(4-bromophenyl)-2-pivalamidoacetate $(400 \mathrm{mg}, 1.2 \mathrm{mmol})$ and 4(hydroxymethyl)phenylboronic acid $(222 \mathrm{mg}, 1.5 \mathrm{mmol})$ in THF $(6 \mathrm{~mL})$ was added $\mathrm{Na}_{2} \mathrm{CO}_{3}(1 \mathrm{M}$, 2.0 eq). A steady stream of nitrogen was bubbled through the mixture for $5 \mathrm{~min}$, before $\mathrm{PdCl}_{2}\left(\mathrm{PPh}_{3}\right)_{2}(0.03 \mathrm{eq})$ was added. The mixture was heated at $100^{\circ} \mathrm{C}$ in a sealed tube for $2 \mathrm{~h}$. After cooling, the mixture was diluted with EtOAc $(10 \mathrm{~mL})$ and water $(10 \mathrm{~mL})$, and the aqueous layer discarded. The organic layer was concentration under reduced pressure and then purified by flash chromatography to give the title compound (385 mg, 89\%) of the title compound. ${ }^{1} \mathrm{H}$ NMR $\delta 7.54$ (d, $J=8.3 \mathrm{~Hz}, 2 \mathrm{H}), 7.50$ (d, J = 8.2 Hz, 2H), $7.40(\mathrm{~d}, J=8.3 \mathrm{~Hz}, 2 \mathrm{H}), 7.38$ (d, $J=8.3 \mathrm{~Hz}, 2 \mathrm{H}), 6.87$ (d, $J=6.8 \mathrm{~Hz}, 1 \mathrm{H}), 5.57(\mathrm{~d}, J=6.8 \mathrm{~Hz}, 1 \mathrm{H}), 4.65(\mathrm{~s}, 2 \mathrm{H}), 3.70(\mathrm{~s}, 3 \mathrm{H}), 1.24(\mathrm{~s}, 9 \mathrm{H}) ;{ }^{13} \mathrm{C}$ NMR $\delta$ $178.1,171.5,141.0,140.6,139.1$, 135.3, 127.5, 127.4, 127.3, 126.9, 64.2, 56.1, 52.7, 38.5, 27.2; LC-MS $t_{\mathrm{R}}: 3.2 \mathrm{~min}, \mathrm{~m} / \mathrm{z} 356.0[\mathrm{MH}]^{+}$.

N-(2-(Hydroxyamino)-1-(4'-(hydroxymethyl)-[1,1'-biphenyl]-4-yl)-2-oxoethyl)pivalamide (3). Methyl 2-(4'-(hydroxymethyl)-[1,1'-biphenyl]-4-yl)-2-pivalamidoacetate (2) (180 mg, $0.51 \mathrm{mmol})$ and $\mathrm{NH}_{2} \mathrm{OH} . \mathrm{HCl}(8.0$ eq) were dissolved in anhydrous $\mathrm{MeOH}$. $\mathrm{KOH}(5 \mathrm{M}$ in $\mathrm{MeOH}, 10$ eq) was added and the reaction mixture was stirred at RT overnight. After evaporation of the solvent, the crude product was purified by flash chromatography (eluent $\mathrm{MeOH} / \mathrm{DCM} 0: 100$ to $10: 90$ ) to give the title compound as a white solid (155 mg, 86\%). ${ }^{1} \mathrm{H}$ NMR (DMSO- $\left.d_{6}\right) \delta 11.00$ (s, $1 \mathrm{H}$ ), 9.05 (s, $1 \mathrm{H}$ ), 7.70 (d, $J=8.0 \mathrm{~Hz}, 1 \mathrm{H}), 7.63(\mathrm{~d}, J=6.3 \mathrm{~Hz}, 2 \mathrm{H}), 7.61(\mathrm{~d}, J=6.2 \mathrm{~Hz}, 2 \mathrm{H}), 7.48(\mathrm{~d}, J=8.3 \mathrm{~Hz}, 2 \mathrm{H}), 7.40$ (d, $J=8.2 \mathrm{~Hz}, 2 \mathrm{H}), 5.40$ (d, J = 8.0 Hz, 1H), 5.20 (t, $J=5.7 \mathrm{~Hz}, 1 \mathrm{H}), 4.53(\mathrm{~d}, J=5.7 \mathrm{~Hz}, 2 \mathrm{H}), 1.16$ $(\mathrm{s}, 9 \mathrm{H}) ;{ }^{13} \mathrm{C}$ NMR (DMSO-d $)$ $\delta 177.0,166.9,141.9,139.6,138.3,138.1,127.5,127.2,126.6,126.5$, 62.8, 53.7, 38.3, 27.3; m/z HRMS (TOF ES ${ }^{+}$) $\mathrm{C}_{20} \mathrm{H}_{25} \mathrm{~N}_{2} \mathrm{O}_{4}[\mathrm{MH}]^{+}$calcd 357.1809; found 357.1818; LC-MS $t_{R}: 3.0 \mathrm{~min}$; HPLC $t_{\mathrm{R}}: 5.0 \mathrm{~min},>99 \%$. 
Aminopeptidase activity assays using recombinant purified protein. Recombinant PfA-M17 (amino acids 84-605) and PfA-M1 (amino acids 85-605) expressed in Escherichia coli and purified by metal affinity chromatography followed by size-exclusion gel filtration was described previously (19). Aminopeptidase activity was assessed by fluorescence assays using the fluorogenic peptide L-leucine-7-amido-4 methylcoumarin hydrochloride (Sigma L2145) as a substrate as previously described (19). Michaelis-Menten constants $\left(K_{\mathrm{m}}\right)$ were calculated for each enzyme purification and showed similar activity as reported previously $(21,36)$. Inhibition of aminopeptidase activity was measured using a Morrison inhibition constant $\left(\mathrm{K}_{\mathrm{i}}^{(\mathrm{app})}\right)$, where enzymes were preincubated in 100 $\mathrm{mM}$ Tris- $\mathrm{HCl}, \mathrm{pH} 8.0$ (supplemented with $2 \mathrm{mM} \mathrm{CoCl} 2$ for PfA-M17) and compound 3 for 10 min prior to the addition of substrate (20 $\mu \mathrm{M}$ for PfA-M1, $10 \mu \mathrm{M}$ for PfA-M17). Substrate concentrations did not exceed the $K_{m}$ for each enzyme. The inhibitor concentration range was selected to obtain a complete inhibition curve $(0-100 \%) . \mathrm{K}_{\mathrm{i}}^{(\mathrm{app})}$ values were calculated by plotting the initial rates versus inhibitor concentration, and fitting to the Morrison equation for tight-binding inhibitors in GraphPad Prism software (nonlinear regression method). Inhibition constants were calculated in biological triplicate from three different protein preparations. $\mathrm{K}_{\mathrm{i}}^{(\mathrm{app})}$ value represents the mean and standard error of the mean (SEM).

Structural Biology. PfA-M17 was co-crystallized with 3 by the hanging-drop method, using previously established protocols (19). Briefly, PfA-M17 was concentrated to $10 \mathrm{mg} / \mathrm{mL}$ and cocrystallized with a final ligand concentration of $1 \mathrm{mM}$ in 30-40\% PEG400, $0.1 \mathrm{M}$ Tris pH 7.5-8.5, $0.2 \mathrm{M} \mathrm{Li}_{2} \mathrm{SO}_{4}$. Crystals were soaked overnight in mother liquor supplemented with $1 \mathrm{mM}$ ligand and $1 \mathrm{mM} \mathrm{ZnSO}_{4}$ before being harvested for data collection. Crystals were snap-frozen in liquid nitrogen, and data were collected $100 \mathrm{~K}$ using synchrotron radiation at the Australian Synchrotron beamlines 3ID1(MX2). Data was processed using XDS and Aimless as part of the CCP4i program suite. The structures were solved by molecular replacement in Phaser RCSB ID 3KQZ as the search model. The structures were refined using Phenix with $5 \%$ of reflections set aside for calculation of Rfree. Between refinement cycles, the protein structure, solvent, and inhibitor were manually built into $2 F O-F C$ and $F O-F C$ electron density maps using COOT with restraint files generated by Phenix where necessary. Data collection and refinement statistics can be found in 
Table S1. The coordinates and structure factors are available from the Protein Data Bank with PDB accession code 7RIE.

Antibody production. Recombinant PfA-M17 (amino acids 84-605;(19)) was used to generate polyclonal rabbit antibodies using The Walter and Eliza Hall Institute of Medical Research Antibody Facility. Briefly, a prebleed was collected from rabbits prior to subcutaneous immunization on four occasions with $200 \mu \mathrm{g}$ of protein, the first in Freund's complete adjuvant and the subsequent immunizations in incomplete adjuvant. The final rabbit bleed (and prebleed for comparison) was used to probe for PfA-M17.

Plasmid constructs. A transgenic P. falciparum line allowing conditional knockdown of PfA-M17 was generated by inserting the last 1017 base pairs of PfA-M17 (Pf3D7_1446200), excluding the stop codon, into the Bglll and Pstl sites of pPfTEX88-HAglmS using oligonucleotides DO733 and DO734 (37). This resulted in the fusion of the PfA-M17 C-terminus with a triple hemagglutinin (HA) and single streptavidin epitope tag. Oligonucleotide sequences are provided in Table S2.

Parasite culture and transfection. P. falciparum 3D7 and transgenic lines were cultured continuously (38) in $\mathrm{O}^{+}$human erythrocytes obtained from the Australian Red Cross at $4 \%$ hematocrit. Complete culturing media contained RPMl 1640 medium (Life Technologies), 20 mg/L gentamicin, $50 \mathrm{mg} / \mathrm{L}$ hypoxanthine, $25 \mathrm{mM}$ sodium bicarbonate, $25 \mathrm{mM}$ HEPES and 0.5\% (w/v) Albumax II (Life Technologies). Minimal media (ResolvingImages) contained the same constituents as complete media except that isoleucine was the only amino acid present. Parasite cultures were maintained at $37^{\circ} \mathrm{C}$ at atmospheric conditions of $5 \% \mathrm{CO}_{2}$ and $1 \% \mathrm{O}_{2}$ in $\mathrm{N}_{2}$. Transfections were performed as previously described (39) and selected for with $2.5 \mathrm{mM} \mathrm{WR99210} \mathrm{(Jacobus).} \mathrm{Parasite}$ cultures were visualized by making thin blood smears on glass slides, which were fixed with methanol for 10 seconds and then stained with Giemsa (1:10 dilution in water; Merck) for $5-10$ min prior to microscopy under $100 \times$ magnification with oil.

\section{Analysis of PfA-M17 expression over the erythrocytic lifecycle and Western blotting.}

Erythrocytes infected with wildtype PF3D7 parasites were heparin synchronized as described (40) and allowed to reinvade for $4 \mathrm{~h}$ before sorbitol synchronization (41) to remove remaining schizonts. 
Parasites were cultured for a cycle and samples were taken at six hourly intervals and lysed in $0.1 \%(\mathrm{w} / \mathrm{v})$ saponin. Parasite lysates were separated on $4-15 \%$ Mini-PROTEAN® TGXTM Gels (Biorad) and transferred to a nitrocellulose membrane for Western blotting. After blocking in 3\% $(\mathrm{w} / \mathrm{v})$ bovine serum albumin (BSA) in PBS, the membrane was incubated with rabbit anti-M17 (1:1000) and rabbit anti-HSP101 (1:1000;(42)) as a loading control. After washing, the blots were incubated with horseradish peroxidase-conjugated secondary antibodies (1:10,000; Thermo Scientific). Protein bands were detected using the Clarity ECI Western blotting substrate (Biorad) and imaged using a Fujifilm LAS-4000 Luminescent Image Analyzer. ImageJ software $(\mathrm{NIH}$, version 151r) was used to measure the intensity of the bands and GraphPad Prism (V.8.4.2) was used to plot the densitometry. Western blotting to confirm expression of epitope tagged PfA-M17 was performed on asynchronous parasite lysates using rabbit anti-M17 (1:1000) and rabbit anti HSP70 (1:1000).

Sequential solubility assay. Erythrocytes infected with Pf3D7 parasites at trophozoite stage were lysed in $0.05 \%(\mathrm{w} / \mathrm{v})$ saponin in PBS containing a complete protease inhibitor cocktail (SigmaAldrich). Sequential solubilization was performed as previously described (43). Briefly, the parasite pellet was resuspended in a hypotonic lysis buffer (1 mM HEPES, pH 7.4) and incubated on ice for 30 min before undergoing three rounds of freeze-thawing in liquid nitrogen. The solution was then centrifuged at $100,000 \mathrm{~g}$ for $30 \mathrm{~min}$ at $2^{\circ} \mathrm{C}$ and the supernatant, containing the soluble proteins, collected for analysis. The pellet was then resuspended in $0.1 \mathrm{M} \mathrm{Na}_{2} \mathrm{CO}_{3}(\mathrm{pH} 11.5)$, incubated for $30 \mathrm{~min}$ and centrifuged, followed by collection of the supernatant fraction, which contained membrane-associated proteins. The remaining pellet was resuspended in $1 \%(\mathrm{v} / \mathrm{v})$ Triton $\mathrm{X}-100$ in PBS and incubated at room temperature before undergoing centrifugation, with the supernatant containing integral membrane proteins. The final pellet, which contained the insoluble proteins, was resuspended in $1 \%(\mathrm{v} / \mathrm{v})$ Triton $\mathrm{X}-100$ in PBS. All samples underwent SDS-PAGE gel electrophoresis and Western blotting, with membranes probed with the following antibodies: rabbit anti-M17 (1:1000), rabbit anti-GAPDH (1:1000), rabbit anti-HSP101 (1:1000(42)) and rabbit anti$\operatorname{EXP2}(1: 1000(44))$. 
Immunofluorescence analysis (IFA). Transgenic parasites were smeared onto glass slides and allowed to dry overnight before being fixed using acetone:methanol $(90: 10)$ for 2 min at $-20^{\circ} \mathrm{C}$. Slides were air dried before being placed at $-20^{\circ} \mathrm{C}$ until analysis. For immunofluorescent assays, slides were thawed at $37^{\circ} \mathrm{C}$ for 10 min and blocked with $1 \%(\mathrm{w} / \mathrm{v}) \mathrm{BSA}$ for $1 \mathrm{~h}$. Primary antibody rat anti-HA (Life Technologies) was diluted 1:250 in 0.5\% BSA and applied to slides for $2 \mathrm{~h}$ before three 5 min washes in PBS. The appropriate AlexaFluor 488-conjugated secondary antibody (1:1000; Life Technologies) was diluted in 0.5\% BSA and incubated on slides for $1 \mathrm{~h}$ before being washed for 5 min three times in PBS. Cover slips were mounted using Prolong Gold Antifade reagent containing 4',6-diamidino-2-phenylindole (DAPI; Life Technologies) and incubated overnight at $37^{\circ} \mathrm{C}$. Images were taken on a Nikon Eclipse Ti2 microscope at $100 \mathrm{x}$ magnification under oil immersion and processed using ImageJ software ( $\mathrm{NIH}$, version 1.53c).

Knockdown of PfA-M17 expression in $P$. falciparum and growth analysis. Heparin synchronized PfM17-HAglmS parasites were treated with $2.5 \mathrm{mM}$ glucosamine (GlcN) at 0-4 h post-invasion; untreated parasites and Pf3D7 parasites treated with $2.5 \mathrm{mM}$ glucosamine served as controls. Parasites were harvested in the first cycle of GlcN treatment (cycle 1) at trophozoite stage, as well as the cycle following (cycle 2) at the same stage. Harvested parasites were lysed in $0.05 \%$ saponin, separated by SDS-PAGE electrophoresis and knockdown was analyzed by Western blotting using mouse anti-HA (1:1000; Sigma) antibody with rabbit anti-EXP2 (1:1000) as a loading control. Assessment of parasite growth \pm GlcN was determined by analysis of Giemsastained smears and images taken with a SC50 5-megapixel or IX71 color camera (Olympus). The parasitemia was determined daily for 6 days following \pm GlcN treatment by counting a minimum of 1000 cells. For each day, the stage of development was also determined and plotted on GraphPad Prism (V.8.4.1). Survival of parasites seeded in 96-well plates at 100 parasites per well was determined after 10 days in culture by Sybr Green I assay. Briefly, after freeze-thawing at $-80^{\circ} \mathrm{C}$, an equal volumes of Lysis buffer (20 mM Tris pH 7.5, 5 mM EDTA, $0.008 \%$ saponin (w/v) \& $0.008 \%$ Triton x-100 (v/v))(45) containing $0.2 \mu \mathrm{L} / \mathrm{mL}$ SYBR Green I Nucleic Acid Gel Stain $(10,000 \mathrm{x}$ in DMSO; ThermoFisher) was added to each well and incubated for $1 \mathrm{~h}$ at RT before fluorescence intensity was read on a Glomax® Explorer Fully Loaded (Promega) with emission wavelengths of 
500-550 nm and an excitation wavelength of $475 \mathrm{~nm}$ and graphs were generated using GraphPad Prism (V.8.4.1). All experiments were performed in 3 biological replicates and unless otherwise stated, a two-tailed unpaired t-test was used herein to determine statistical significance, with data presented as the mean and error bars representative of standard deviation.

Hemozoin Assay. Erythrocytes infected with PfM17-HAglmS or Pf3D7 underwent sorbitol synchronization before cultures were treated \pm GlcN and incubated for a further cycle until parasites developed into trophozoites. Once parasites reached developmentally similar stages, pellets were resuspended in $800 \mu \mathrm{L}$ of $2.5 \%$ SDS in $0.1 \mathrm{M}$ sodium bicarbonate $\mathrm{pH} 8.8$, and then incubated at RT with rotation for $20 \mathrm{~min}$, before undergoing centrifugation at $13,000 \mathrm{~g}$ for $10 \mathrm{~min}$. Each pellet was then washed twice with $1 \mathrm{~mL}$ of the same solution before being resuspending in $500 \mu \mathrm{L}$ of $5 \%$ SDS, $50 \mathrm{mM} \mathrm{NaOH}$ and incubating for a further $20 \mathrm{~min}$ with rotation. The quantity of monomeric heme was then measured at 405 nM on a Glomax® Explorer Fully Loaded (Promega). Three or four biological replicates were performed, and the data plotted using GraphPad Prism 9 where significance was determined by a two-tailed unpaired t-test. Parasites were assessed for multiple DVs via Geimsa staining and under $100 \times$ magnification with oil, with a combined $\geq 200$ parasites counted from 4 biological replicates for each treatment group. A Kruskal-Wallis test with Dunn's post hoc multiple comparison test was used to determine the significance.

Determination of compound $\mathbf{E C}_{50}$. Parasite viability assays were adapted from previously described methods (46). Briefly, sorbitol synchronized ring stage Pf3D7 parasites were cultured in 96-well U-bottom plates at $0.5 \%$ parasitemia and $2 \%$ hematocrit, to which $50 \mu \mathrm{L}$ of serially diluted 3, artemisinin or artesunate (Sigma) was added. After $72 \mathrm{~h}$ under standard culturing conditions, plates were placed at $-80^{\circ} \mathrm{C}$ before analysis using the SYBR Green I assay as described above. Uninfected RBCs and parasites treated with the vehicle control DMSO were used to normalize fluorescence. Data from 3 or 4 biological replicates performed in triplicate was plotted as 4parameter log dose nonlinear regression analysis with a sigmoidal dose-response curve fitted using GraphPad Prism 9 to generate the $\mathrm{EC}_{50}$ values, with error bars representative of the SEM. 
Parasite killing rate assay: Assay was performed as previously described with some alterations (47). Briefly, sorbitol synchronized ring or trophozoite stage parasites were cultured in the presence of $10 \mathrm{x}$ the $\mathrm{EC}_{50}$ of $\mathbf{3}$ or artesunate for either 24 or $48 \mathrm{~h}$. Cultures that were incubated with either compound for $48 \mathrm{~h}$ were fed at $24 \mathrm{~h}$ with fresh media containing $10 \mathrm{x}$ the $\mathrm{EC}_{50}$ of compound. At the completion of these times, RBCs were thoroughly washed to remove the compound and cultures were diluted $1 / 3$ with fresh media and further grown for $48 \mathrm{~h}$ before aliquots were placed at $-80^{\circ} \mathrm{C}$. Cultures were thawed and analyzed using SYBR Green I assay as described above. Parasite viability was determined as a percentage of DMSO-treated parasites cultured alongside compound treated parasites. Artesunate was used as a positive control and for comparison of the parasite killing rate, and experiments were performed in 4 biological replicates.

Sample preparation for metabolomics analysis. For experiment 1 (Fig. 7) and 2 (Fig. S4), heparin synchronized Pf3D7 and PfM17-HAglmS parasites were allowed to invade RBC for $4 \mathrm{~h}$ and any remaining schizonts were lysed by sorbitol synchronization. For experiment 1, parasite cultures were then treated for $\sim 36 \mathrm{~h}$ with $2.5 \mathrm{mM} \mathrm{GlcN}$ or for $1 \mathrm{~h}$ with 3 at $10 \mathrm{x}$ the $\mathrm{EC}_{50}$ (Pf3D7 only) or left untreated, while for experiment 2, parasite cultures were only treated with GlcN. Parasites were harvested at developmentally similar timepoints by centrifugation at $900 \mathrm{~g}$ for $5 \mathrm{~min}$ and then resuspended in $10 \mathrm{~mL}$ of chilled PBS. Parasite metabolism was quenched by cooling samples to between $3-5^{\circ} \mathrm{C}$ in an ethanol-dry ice bath. The rest of the preparation was performed at $4^{\circ} \mathrm{C}$. Parasites were magnet purified on a VarioMACS column and $3 \times 10^{7}$ parasites were used for downstream analysis. For experiment 3 (Fig. S5), Pf3D7 cultures underwent double sorbitol synchronization $14 \mathrm{~h}$ apart, followed by further incubation for $28-42 \mathrm{~h}$ to achieve the desired trophozoite stage $(28 \mathrm{hpi})$ at $6 \%$ parasitaemia and $2 \%$ hematocrit. Infected RBCs $\left(2 \times 10^{8}\right)$ were treated with $10 \mathrm{x}$ the $\mathrm{EC}_{50}$ of 3 for $1 \mathrm{~h}$, after which metabolites were extracted. All samples (from experiment 1, 2 and 3) were centrifuged at $650 \mathrm{~g}$ for $3 \mathrm{~min}$, the supernatant was removed, and the pellet washed in $500 \mu \mathrm{L}$ of ice-cold PBS. Samples were again centrifuged at $650 \mathrm{~g}$ for $3 \mathrm{~min}$ and pellets were resuspended in $150 \mu \mathrm{L}$ of ice-cold extraction buffer (100\% methanol) and quickly resuspended. The samples were then incubated on a vortex mixer for $1 \mathrm{~h}$ at $4^{\circ} \mathrm{C}$ before being centrifuged at $17,000 \mathrm{~g}$ for $10 \mathrm{~min}$; from this $100 \mu \mathrm{L}$ of supernatant was collected and stored at - 
$80^{\circ} \mathrm{C}$ until analysis. For each sample, another $10 \mu \mathrm{L}$ was collected and pooled, to serve as a quality control (QC) sample.

Liquid chromatography- mass spectrometry (LC-MS) analysis. Liquid chromatography-mass spectrometry (LC-MS) data was acquired on a Q-Exactive Orbitrap mass spectrometer (Thermo Scientific) coupled with high-performance liquid chromatography system (HPLC, Dionex Ultimate® 3000 RS, Thermo Scientific) as described previously (48). Briefly, chromatographic separation was performed on ZIC-pHILIC column equipped with a guard (5 $\mu \mathrm{m}, 4.6 \times 150 \mathrm{~mm}$, SeQuant®, Merck). The mobile phase (A) was $20 \mathrm{mM}$ ammonium carbonate (Sigma Aldrich), (B) acetonitrile (Burdick and Jackson) and needle wash solution was $50 \%$ isopropanol. The column flow rate was maintained at $0.3 \mathrm{~mL} / \mathrm{min}$ with temperature at $25{ }^{\circ} \mathrm{C}$ and the gradient program was: $80 \%$ B to $50 \%$ B over $15 \mathrm{~min}$, then to $5 \% \mathrm{~B}$ at $18 \mathrm{~min}$ until $21 \mathrm{~min}$, increasing to $80 \% \mathrm{~B}$ at $24 \mathrm{~min}$ until $32 \mathrm{~min}$. Total run time was 32 min with an injection volume of $10 \mu \mathrm{L}$. Mass spectrometer was operated in full scan mode with positive and negative polarity switching at 35,000 resolution at $200 \mathrm{~m} / \mathrm{z}$ with detection range of 85 to $1275 \mathrm{~m} / \mathrm{z}$, AGC target was $1 \mathrm{e} 6$ ions with a maximum injection time of 50 ms. Electro-spray ionization source (ESI) was set to $4.0 \mathrm{kV}$ voltage for positive and negative mode, sheath gas was set to 50 , aux gas to 20 and sweep gas to 2 arbitrary units, capillary temperature $300^{\circ} \mathrm{C}$, probe heater temperature $120^{\circ} \mathrm{C}$. Approximately 350 authentic metabolite standards were analyzed at the start of each batch to provide accurate retention times to facilitate metabolite identification. Metabolomics samples from each experiment were analyzed as single batches in random order with periodic injections of the pooled QC, and blank samples, to assess analytical quality and aid downstream metabolite identification procedures.

Metabolomics LC-MS data processing. The acquired LCMS data was processed in untargeted fashion using open source software, IDEOM (49) (http:// mzmatch.sourceforge.net/ideom.php) using an updated metabolite database containing all peptides of proteinergic amino acids up to 5 amino acids in length. Initially, ProteoWizard was used to convert raw LC-MS files to $m z X M L$ format and XCMS (Centwave) to pick peaks and convert to peakML files. Mzmatch. $R$ was used for alignment and annotation of related metabolite peaks with a minimum detectable intensity of 
100,000 , relative standard deviation (RSD) of $<0.5$ (reproducibility), and peak shape (codadw) of $>0.8$. Default IDEOM parameters were used to eliminate unwanted noise and artefact peaks. Loss or gain of a proton was corrected in negative and positive ESI mode, respectively, followed by putative identification of metabolites by accurate mass within 3 ppm mass error searching against the IDEOM metabolite database. To reduce the number of false positive identifications, retention time error was calculated for each putative ID using IDEOM built-in retention time model which uses actual retention time data of authentic standards ( 350 standards). Principal-component analysis (PCA) and hierarchical clustering algorithms were performed in Metaboanalyst (50). Metabolomics data are presented as relative abundance values from 3 technical replicates for experiment 1 and 2, while for experiment 3, data is from 4-9 biological replicates. Differences were determined using Welch's $t$ test where significant interactions were observed. Significance was determined at $p$ values $<0.05$. To assess whether identified peptides could be derived from hemoglobin, the sequences of human hemoglobin $\alpha, \beta$ and $\delta$ chains (HBA_HUMAN P69905, HBB_HUMAN P68871, HBD_HUMAN P02042) were searched for any peptide with monoisotopic mass within $0.002 \mathrm{~m} / \mathrm{z}$ of the identified peptide using custom Python scripts.

\section{Acknowledgments}

We thank the Australian Red Cross for providing red blood cells used in this study. We would also like to thank Professor Susan Charman and the Centre for Drug Candidate Optimisation (CDCO) team for providing compound analysis. R.C.S.E. and T.R.M. were recipients of an Australian Government Research Training Program Stipend. This work was supported by an NHMRC Synergy Grant (1185354). T.F.dK.-W is the recipient of an NHMRC Fellowship (1136300).

Author Contributions: R.C.S.E. conceptualized the study, performed experiments, analyzed data, and wrote the paper. G.S. performed experiments, analyzed data, and wrote the paper. K.H created the transgenic parasite line. N.A.C performed experiments, analyzed data, supervised 
work, and wrote the paper. T.R.M. performed experiments and C.T.W. analyzed data. P.J.S. designed the compound, supervised work, and wrote the paper. N.B.V. synthesized the compound and contributed to its design. C.A.M. analyzed data and wrote the paper. D.C. supervised work and wrote the paper. S.M. conceptualized the study, performed experiments, analyzed data, supervised work, and wrote the paper. T.F.dK.-W conceptualized the study, performed experiments, analyzed data, supervised work and wrote the paper. All authors edited the final paper.

Competing Interest Statement: The authors declare no conflict of interest.

\section{References}

1. WHO (2020) World malaria report 2020. (World Health Organisation, Geneva), p 299.

2. Miotto O, et al. (2020) Emergence of artemisinin-resistant Plasmodium falciparum with kelch13 C580Y mutations on the island of New Guinea. PLoS Pathog. 16(12):e1009133.

3. Uwimana A, et al. (2020) Emergence and clonal expansion of in vitro artemisininresistant Plasmodium falciparum kelch13 R561H mutant parasites in Rwanda. Nat. Med. 26(10):1602-1608.

4. Uwimana A, et al. (2021) Association of Plasmodium falciparum kelch13 R561H genotypes with delayed parasite clearance in Rwanda: an open-label, single-arm, multicentre, therapeutic efficacy study. Lancet Infect Dis 21(8):1120-1128.

5. Ataide R, et al. (2017) Host immunity to Plasmodium falciparum and the assessment of emerging artemisinin resistance in a multinational cohort. PNAS 114(13):3515-3520.

6. Babbitt SE, et al. (2012) Plasmodium falciparum responds to amino acid starvation by entering into a hibernatory state. PNAS 109(47):E3278.

7. Krugliak M, Zhang J, \& Ginsburg H (2002) Intraerythrocytic Plasmodium falciparum utilizes only a fraction of the amino acids derived from the digestion of host cell cytosol for the biosynthesis of its proteins. Mol. Biochem. Parasitol. 119(2):249-256.

8. Rosenthal PJ (2002) Hydrolysis of erythrocyte proteins by proteases of malaria parasites. Curr. Opin. Hematol. 9(2):140-145.

9. Lew VL, Macdonald L, Ginsburg H, Krugliak M, \& Tiffert T (2004) Excess haemoglobin digestion by malaria parasites: a strategy to prevent premature host cell lysis. Blood Cells Mol. Dis. 32:353-359. 
10. Liu J, Istvan ES, Gluzman IY, Gross J, \& Goldberg DE (2006) Plasmodium falciparum ensures its amino acid supply with multiple acquisition pathways and redundant proteolytic enzyme systems. PNAS 103(23):8840-8845.

11. Bakar NA, Klonis N, Hanssen E, Chan C, \& Tilley L (2010) Digestive-vacuole genesis and endocytic processes in the early intraerythrocytic stages of Plasmodium falciparum. $J$ Cell Sci 123(3):441-450.

12. Goldberg DE (2005) Hemoglobin Degradation. Malaria: Drugs, Disease and Postgenomic Biology, eds Compans RW, Cooper MD, Honjo T, Koprowski H, Melchers F, Oldstone MBA, Olsnes S, Potter M, Vogt PK, Wagner H, et al. (Springer Berlin Heidelberg, Berlin, Heidelberg), pp 275-291.

13. Gavigan CS, Dalton JP, \& Bell A (2001) The role of aminopeptidases in haemoglobin degradation in Plasmodium falciparum-infected erythrocytes. Mol Biochem Parasitol 117:37-48.

14. Nankya-Kitaka MF, Curley GP, Gavigan CS, Bell A, \& Dalton JP (1998) Plasmodium chabaudi chabaudi and $P$. falciparum: inhibition of aminopeptidase and parasite growth by bestatin and nitrobestatin. Parasitol. Res. 84(7):552-558.

15. Gardiner DL, Trenholme KR, Skinner-Adams TS, Stack CM, \& Dalton JP (2006) Overexpression of leucyl aminopeptidase in Plasmodium falciparum parasites: target for the antimalarial activity of bestatin. J. Biol. Chem 281(3):1741-1745.

16. Harbut MB, et al. (2011) Bestatin-based chemical biology strategy reveals distinct roles for malaria M1- and M17-family aminopeptidases. PNAS 108(34):E526-E534.

17. Skinner-Adams TS, et al. (2012) The aminopeptidase inhibitor CHR-2863 is an orally bioavailable inhibitor of murine malaria. Antimicrob. Agents Chemother. 56(6):3244-3249.

18. Mistry SN, et al. (2014) Two-pronged attack: dual inhibition of Plasmodium falciparum M1 and M17 metalloaminopeptidases by a novel series of hydroxamic acid-based inhibitors. J. Med. Chem 57(21):9168-9183.

19. Vinh NB, et al. (2019) Hydroxamic acid inhibitors provide cross-species inhibition of Plasmodium M1 and M17 aminopeptidases. J. Med. Chem 62(2):622-640.

20. Dalal S \& Klemba M (2007) Roles for two aminopeptidases in vacuolar hemoglobin catabolism in Plasmodium falciparum. in J. Biol. Chem (American Society for Biochemistry and Molecular Biology Inc, Bethesda; USA), pp 35978-35987.

21. McGowan S, et al. (2010) Structure of the Plasmodium falciparum M17 aminopeptidase and significance for the design of drugs targeting the neutral exopeptidases. PNAS 107(6):2449.

22. Mathew R, et al. (2021) Biochemical and cellular characterisation of the Plasmodium falciparum M1 alanyl aminopeptidase (PfM1AAP) and M17 leucyl aminopeptidase (PfM17LAP). Sci. Rep. 11(1):2854. 
23. Shafik SH, et al. (2020) The natural function of the malaria parasite's chloroquine resistance transporter. Nat. Commun. 11(1):3922.

24. Hill RJ, Konigsberg W, Guidotti G, \& Craig LC (1962) The structure of Human hemoglobin: I. The separation of the $\alpha$ and $\beta$ chains and their amino acid composition. $J$. Biol. Chem 237(5):1549-1554.

25. Poreba M, et al. (2012) Fingerprinting the substrate specificity of M1 and M17 aminopeptidases of human malaria, Plasmodium falciparum. PLoS ONE 7(2):e31938e31938.

26. Martin RE \& Kirk K (2007) Transport of the essential nutrient isoleucine in human erythrocytes infected with the malaria parasite Plasmodium falciparum. Blood 109(5):2217-2224.

27. Zhang M, et al. (2018) Uncovering the essential genes of the human malaria parasite Plasmodium falciparum by saturation mutagenesis. Science 360(6388):e7847.

28. Lin JW, et al. (2015) Replication of Plasmodium in reticulocytes can occur without hemozoin formation, resulting in chloroquine resistance. J. Exp. Med 212(6):893-903.

29. Zheng J, Jia H, \& Zheng Y (2015) Knockout of leucine aminopeptidase in Toxoplasma gondii using CRISPR/Cas9. Int. J. Parasitol. 45(2-3):141-148.

30. Prommana P, et al. (2013) Inducible knockdown of Plasmodium Gene expression using the glmS ribozyme. PLoS ONE 8(8):e73783.

31. Drinkwater N, et al. (2016) Potent dual inhibitors of Plasmodium falciparum M1 and M17 aminopeptidases through optimization of S1 pocket interactions. Eur. J. Med. Chem. 110:43-64.

32. Giannangelo C, et al. (2020) System-wide biochemical analysis reveals ozonide antimalarials initially act by disrupting Plasmodium falciparum haemoglobin digestion. PLoS Pathog 16(6):e1008485.

33. Prasad R, et al. (2013) Blocking Plasmodium falciparum Development via Dual Inhibition of Hemoglobin Degradation and the Ubiquitin Proteasome System by MG132. PLOS ONE 8(9):e73530.

34. Rosenthal PJ, McKerrow JH, Aikawa M, Nagasawa H, \& Leech JH (1988) A malarial cysteine proteinase is necessary for hemoglobin degradation by Plasmodium falciparum. J. Clin. Investig 82(5):1560-1566.

35. Malcolm TR, et al. (2021) Mapping the substrate specificity of the Plasmodium M1 and M17 aminopeptidases. Biochem. J. 478(13):2697-2713.

36. McGowan S, et al. (2009) Structural basis for the inhibition of the essential Plasmodium falciparum M1 neutral aminopeptidase. PNAS 106(8):2537-2542. 
37. Chisholm SA, et al. (2016) Contrasting inducible knockdown of the auxiliary PTEX component PTEX88 in $P$. falciparum and $P$. berghei unmasks a role in parasite virulence. PLOS ONE 11(2):e0149296.

38. Trager W \& Jensen JB (1976) Human malaria parasites in continuous culture. Science 193:673-675.

39. Fidock DA \& Wellems TE (1997) Transformation with human dihydrofolate reductase renders malaria parasites insensitive to WR99210 but does not affect the intrinsic activity of proguanil. Proc Natl Acad Sci USA 94(20):10931-10936.

40. Boyle MJ, et al. (2010) Isolation of viable Plasmodium falciparum merozoites to define erythrocyte invasion events and advance vaccine and drug development. PNAS 107(32):14378.

41. Lambros C \& Vanderberg JP (1979) Synchronization of Plasmodium falciparum erythrocytic stages in culture. J. Parasitol. 65(3):418-420.

42. de Koning-Ward TF, et al. (2009) A newly discovered protein export machine in malaria parasites. Nature 459(7249):945-949.

43. Counihan NA, et al. (2017) Plasmodium falciparum parasites deploy RhopH2 into the host erythrocyte to obtain nutrients, grow and replicate. Elife 6:e23217.

44. Bullen HE, et al. (2012) Biosynthesis, localization, and macromolecular arrangement of the Plasmodium falciparum translocon of exported proteins (PTEX). J. Biol. Chem 287(11):7871-7884.

45. Smilkstein M, Sriwilaijaroen N, Kelly JX, Wilairat P, \& Riscoe M (2004) Simple and inexpensive fluorescence-based technique for high-throughput antimalarial drug screening. Antimicrob. Agents Chemother. 48(5):1803.

46. Dery V, et al. (2015) An improved SYBR Green-1-based fluorescence method for the routine monitoring of Plasmodium falciparum resistance to anti-malarial drugs. Malar. $J$ 14(1):481.

47. Gilson PR, et al. (2019) Evaluation of 4-Amino 2-Anilinoquinazolines against Plasmodium and Other Apicomplexan Parasites In Vitro and in a $P$. falciparum Humanized NOD-scid IL2Ry(null) Mouse Model of Malaria. Antimicrob. Agents Chemother. 63(3):e0180401818.

48. Creek DJ, et al. (2016) Metabolomics-Based Screening of the Malaria Box Reveals both Novel and Established Mechanisms of Action. Antimicrobial agents and chemotherapy 60(11):6650-6663.

49. Creek DJ, Jankevics A, Burgess KE, Breitling R, \& Barrett MP (2012) IDEOM: an Excel interface for analysis of LC-MS-based metabolomics data. Bioinformatics (Oxford, England) 28(7):1048-1049. 
bioRxiv preprint doi: https://doi.org/10.1101/2021.11.23.469631; this version posted November 23, 2021. The copyright holder for this preprint (which was not certified by peer review) is the author/funder. All rights reserved. No reuse allowed without permission.

50. Chong J, et al. (2018) MetaboAnalyst 4.0: towards more transparent and integrative metabolomics analysis. Nucleic Acids Res. 46(W1):W486-W494. 
A

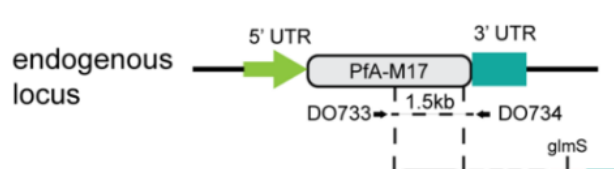

targeting

construct

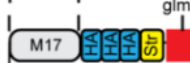

pM17-HAgImS

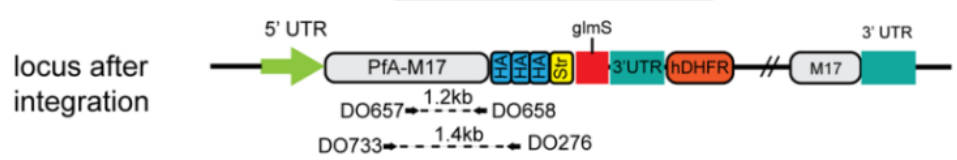

B

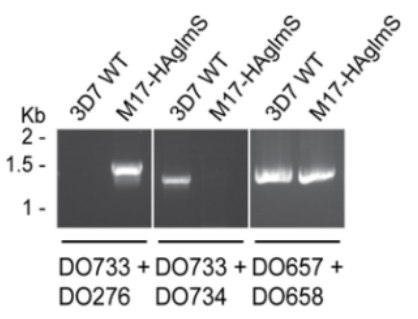

C

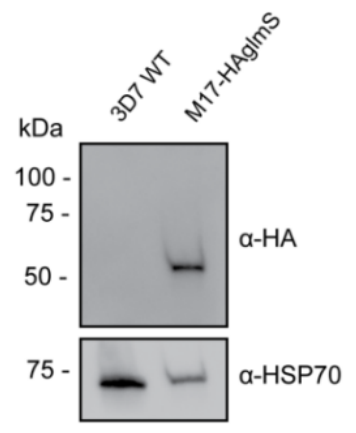

Figure 1. Generation of HA-tagged PfA-M17-HAglmS transgenic parasites. A) Schematic of the Pfa-m17 locus, and locus after single crossover recombination with pM17-HAglmS. The pM17HAglmS plasmid contained the last kilobase of the coding sequence excluding the stop codon fused in frame to $3 \times$ haemagglutinin (HA) and a single strep II (Str) tag. The plasmid also includes a g/mS ribozyme with a synthetic untranslated region (UTR) and the selectable marker human dihydrofolate reductase (hDHFR). Arrows indicate oligonucleotides used in diagnostic PCRs as well as their expected sizes. (B) Diagnostic PCR showing integration of pM17-HAglmS at the endogenous locus. PCR was performed using the oligonucleotide pairs outlined in (A) on DNA extracted from parasites before (Pf3D7) or after (PfA-M17-HAglmS) transfection with the targeting construct. Oligonucleotides DO657 and DO658, which recognize the endogenous locus, serves as a positive control. (C) Western blot analysis of parasite lysates confirming HA expression. The predicted molecular mass of PfA-M17-HA is $72 \mathrm{kDa}$, and HSP70 serves as a loading control. 

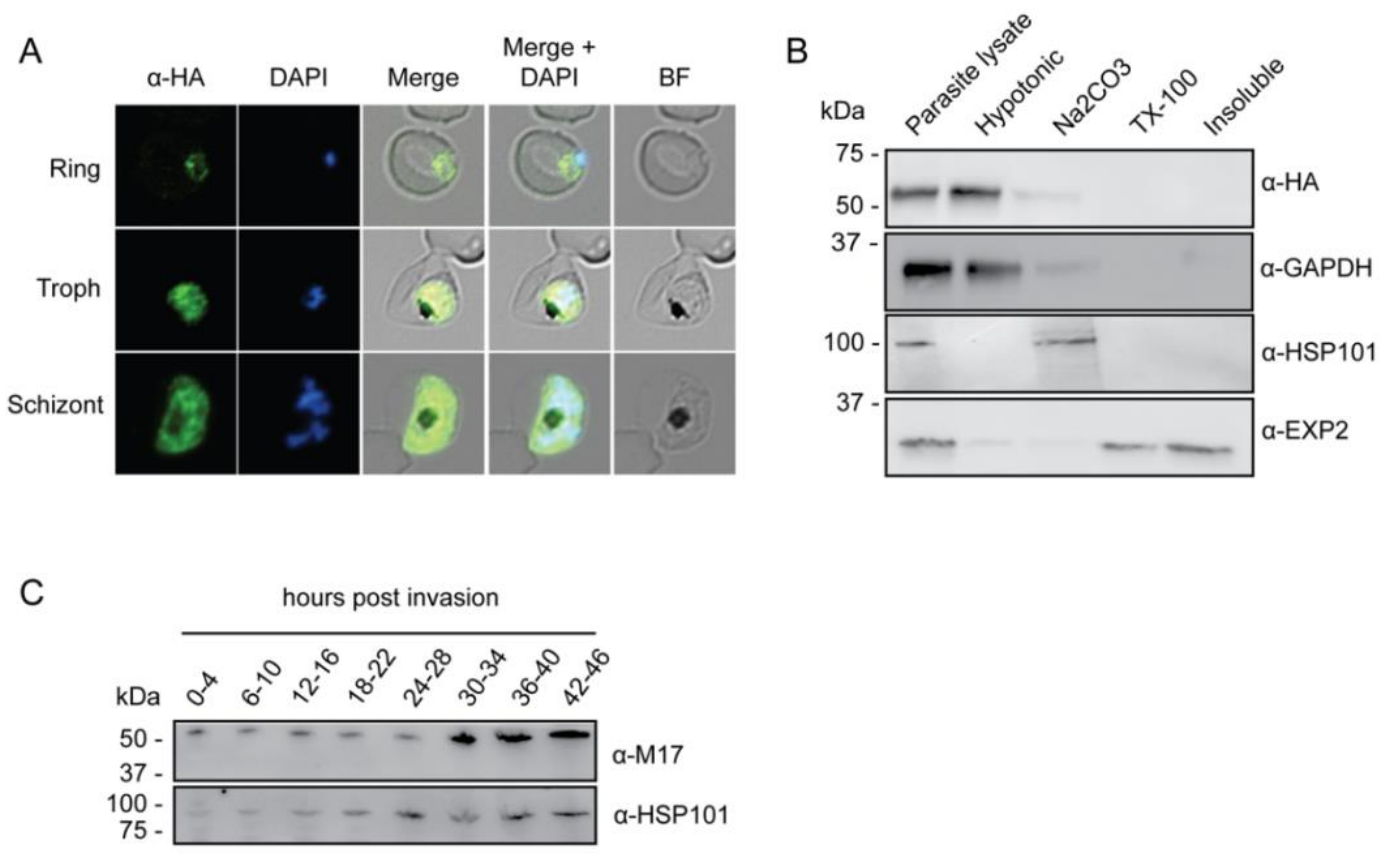

Figure 2. Analysis of PfA-M17 localization and expression over the lifecycle (A) Immunofluorescent analysis (IFA) of PfA-M17-HAglmS parasites in the three distinct lifecycle stages fixed with 90:10 acetone:methanol and probed with anti-HA and DAPI. (B) Saponin-lysed trophozoite stage PfAM17-HAglmS parasites were sequentially lysed in the buffers indicated from left to right and analyzed by Western blotting. Insoluble material represents the remaining pellet after lysis in $1 \%$ Triton X-100. GAPDH, HSP101 and EXP2 serve as controls for cytoplasmic, membraneassociated, and integral membrane proteins respectively. (C) Western blot analysis of endogenous PfA-M17 expression in Pf3D7 wildtype parasites over the erythrocytic cycle probed with anti-M17 antibodies. HSP101 serves as a loading control. 
A
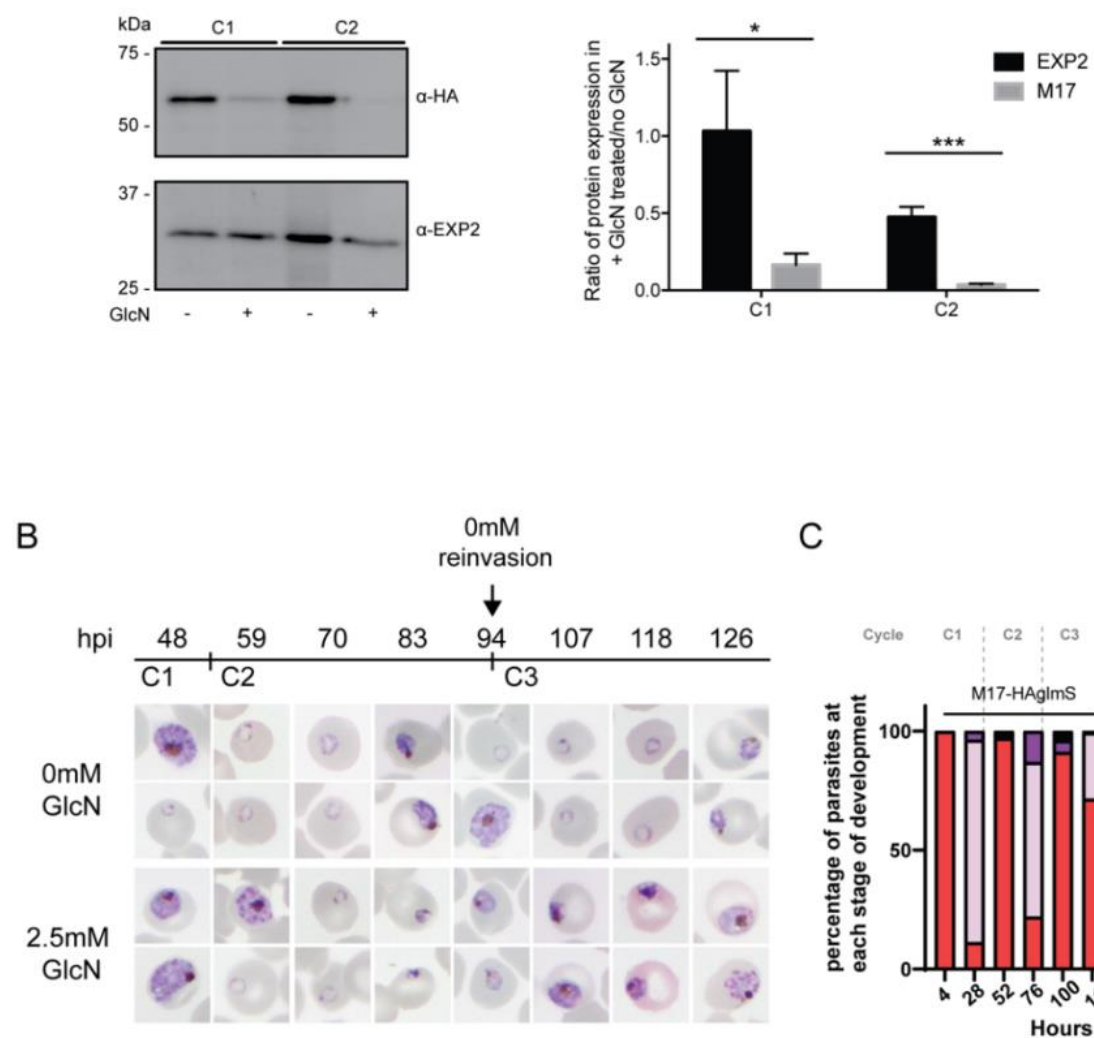

C

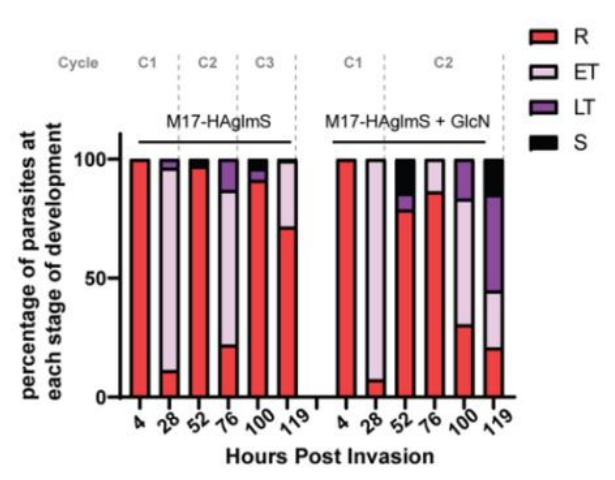

D
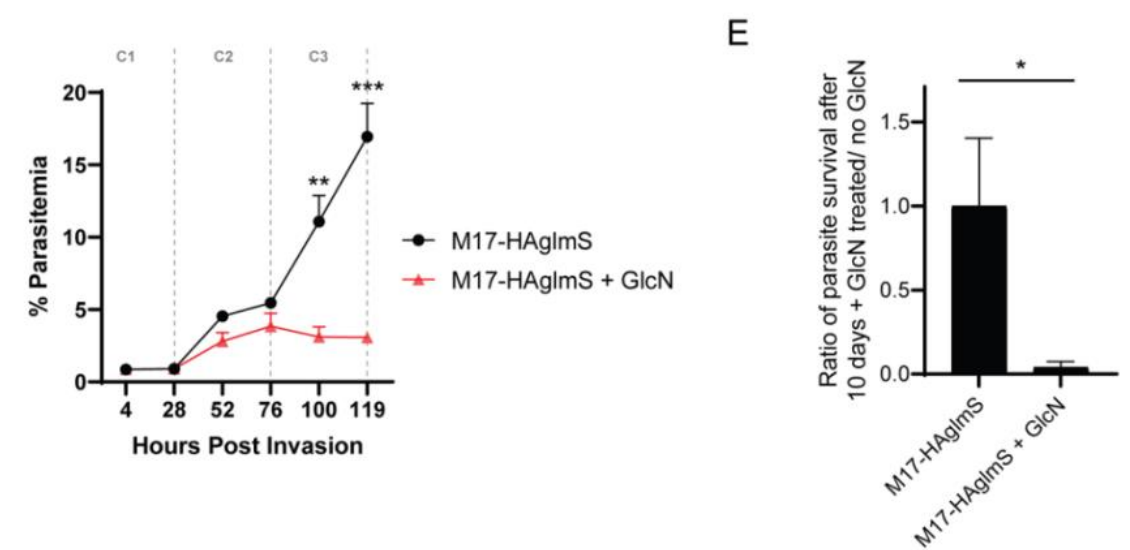

Figure 3. Depletion of PfA-M17 expression leads to perturbed parasite growth in vitro. (A) Knockdown of PfA-M17 expression. Left panel: Representative Western blot of PfA-M17-HAglmS protein lysates prepared from parasites treated with either $2.5 \mathrm{mM} \mathrm{GlcN} \mathrm{(+)} \mathrm{or} \mathrm{left} \mathrm{untreated} \mathrm{(-)} \mathrm{at}$ trophozoite stage in cycle $1(\mathrm{C} 1)$ or cycle 2 (C2). EXP2 serves as a loading control. Right panel: Densitometry of bands observed in Western blots was performed using ImageJ to calculate the 
ratio of PfA-M17 protein expression in GlcN-treated parasites relative to EXP2 compared to that of untreated parasites. Shown is the mean \pm standard deviation $(n=3)$. Statistical significance was determined using an unpaired t-test, ${ }^{*} p \leq 0.05,{ }^{* * *} p \leq 0.001$. (B) Representative Giemsa-stained parasite smears of PfA-M17-HAglmS cultures treated with $0 \mathrm{mM}$ or $2.5 \mathrm{mM}$ GlcN shows depletion of PfA-M17 protein results in a growth delay following reinvasion into cycle 2 (C2). (C) Percentage of PfA-M17-HAglmS parasites at each stage of development \pm GlcN over 3 cycles shows depletion of PfA-M17 leads to delayed parasite development within cycle 2 ( $n=3$ biological replicates) (D) Parasitemias of PfA-M17-HAglmS parasites cultured \pm GlcN over 3 cycles. Invasion into cycle 2 is not significantly affected but the growth delay of GlcN-treated parasites observed in this cycle affects parasitemia thereafter, with a significant difference in parasitemia by the time untreated PfAM17-HAglms have entered cycle 3. Grey dotted lines are representative of the time when untreated parasites have completed a cycle. Plotted is the mean \pm standard deviation $(n=3)$, with statistical significance determined using an unpaired t-test $\left({ }^{* *} p \leq 0.01,{ }^{* * *} p \leq 0.001\right)$. (E) Ratio of parasite survival of PfA-M17-HAglmS after treatment with GlcN for 10 days compared to untreated parasites as determined by Sybr Green 1 assay. Shown is the mean \pm standard deviation $(n=3)$. Statistical significance was determined using an unpaired t-test ( $\left.{ }^{*} p \leq 0.05\right)$. 
A

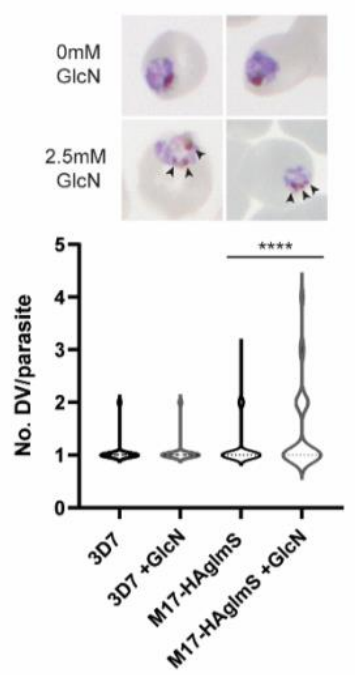

B

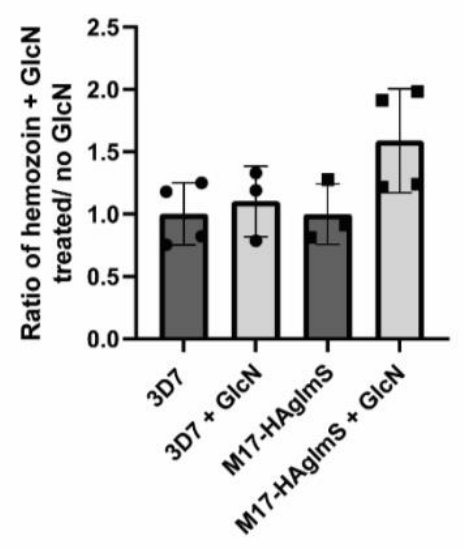

Figure 4. Parasites depleted of PfA-M17 develop significantly more digestive vacuoles the cycle following protein knockdown but hemozoin amount does not change. (A) Upper panel: Representative Giemsa-stained smears of PfA-M17-HAglmS \pm GlcN; black arrows indicate multiple digestive vacuoles. Lower panel: Number of digestive vacuoles per Pf3D7 and PfA-M17-HAglmS the cycle following addition of glucosamine $(\mathrm{GlcN})$ as determined under Giemsa-staining. Shown is combined data from 4 biological replicates $(n \geq 100)$. Statistical significance was determined by a Kruskal-Wallis test with Dunn's post hoc multiple comparison test $\left({ }^{* * *} p \leq 0.0001\right)$. (B) Ratio of hemozoin of Pf3D7 and PfA-M17-HAglmS GlcN-treated parasites relative to untreated parasites. Shown is the mean \pm standard deviation ( $n \geq 3$ ). No statistical significance was determined using an unpaired t-test. 

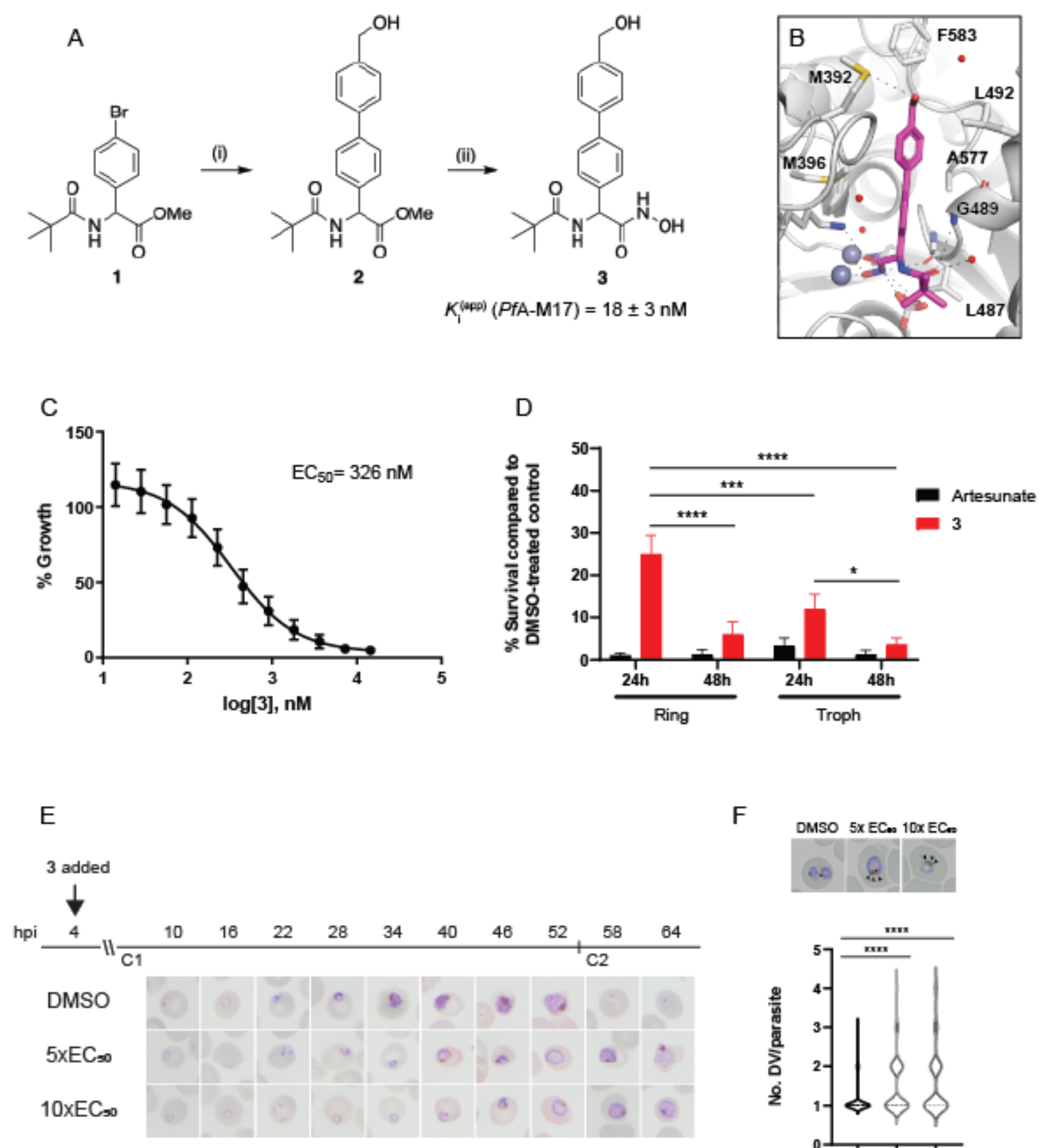

$\mathrm{F}$
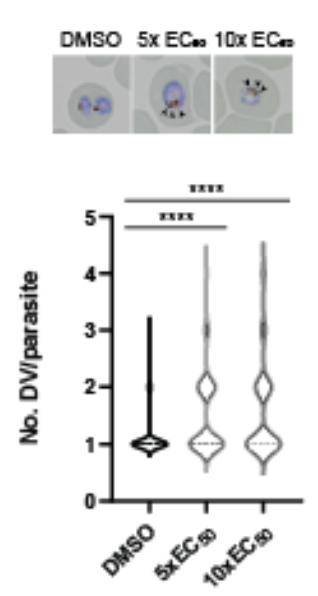

Figure 5. Synthesis and activity of 3, a specific PfA-M17 inhibitor. (A) Scheme 1. Synthesis of 3: (i) Boronic acid, $\mathrm{Pd}\left(\mathrm{PPh}_{3}\right)_{2} \mathrm{Cl}_{2}, \mathrm{Na}_{2} \mathrm{CO}_{3}, \mathrm{THF}, 100^{\circ} \mathrm{C}, 2 \mathrm{~h}$, (ii) $\mathrm{NH}_{2} \mathrm{OH} . \mathrm{HCl}, \mathrm{KOH}, \mathrm{RT}, 16$ h. Inhibition constant for 3 toward recombinant, purified PfA-M17 is shown. (B) Binding mode of $\mathbf{3}$ bound to PfAM17. Solvent-accessible surface of PfA-M17 (grey) with active site ions shown in grey spheres. Stick representation (magenta) shows the binding positions of $\mathbf{3}$. Molecular interactions between $\mathbf{3}$ and PfA-M17 are indicated by dashed lines; water molecules are represented by red spheres. (C) 
Killing action of 3 over $72 \mathrm{~h}$ as determined by SYBR Green I assay. The EC 50 value was calculated from 4 biological replicates performed in triplicate and data plotted as the mean \pm standard error of the mean. (D) Parasite killing rate was determined by incubating Pf3D7 parasites in $10 \times E_{50}$ as previously determined for either 24 or 48 hours before the drug was washed off and parasites allowed to grow for a further 48 hours. Survival was determined via Sybr Green I assay and compared to vehicle (DMSO)-treated controls. Shown is the mean \pm standard deviation $(n=4)(E)$ Synchronized parasites at 4 hours post-invasion (hpi) were treated over two cycles $(\mathrm{C} 1$, cycle 1 ; $\mathrm{C} 2$, cycle 2) with either $5 \mathrm{x}$ or $10 x \mathrm{EC}_{50}$ or $\mathrm{DMSO}$ at the concentration present in the $10 \mathrm{x} \mathrm{EC}_{50}$ treatment. Representative Giemsa-stained smears from 2 biological replicates show delay in parasite maturation to schizogony $\left(5 \times \mathrm{EC}_{50}\right)$ or trophozoite stage $\left(10 \mathrm{x} \mathrm{EC}_{50}\right)$. (F) Upper panel: Representative Giemsa-stained smears of Pf3D7 + DMSO, 5x or 10x the $\mathrm{EC}_{50}$ of 3; black arrows indicate multiple digestive vacuoles. Lower panel: Number of digestive vacuoles per Pf3D7 following the addition of $\mathbf{3}$ at 4 hours post invasion (hpi) as determined under Giemsa-staining. Shown is the combined data from 2 biological replicates $(n \geq 100)$. Statistical significance was determined by a Kruskal-Wallis test with Dunn's post hoc multiple comparison test $\left({ }^{* * * *} p \leq 0.0001\right)$. 
A
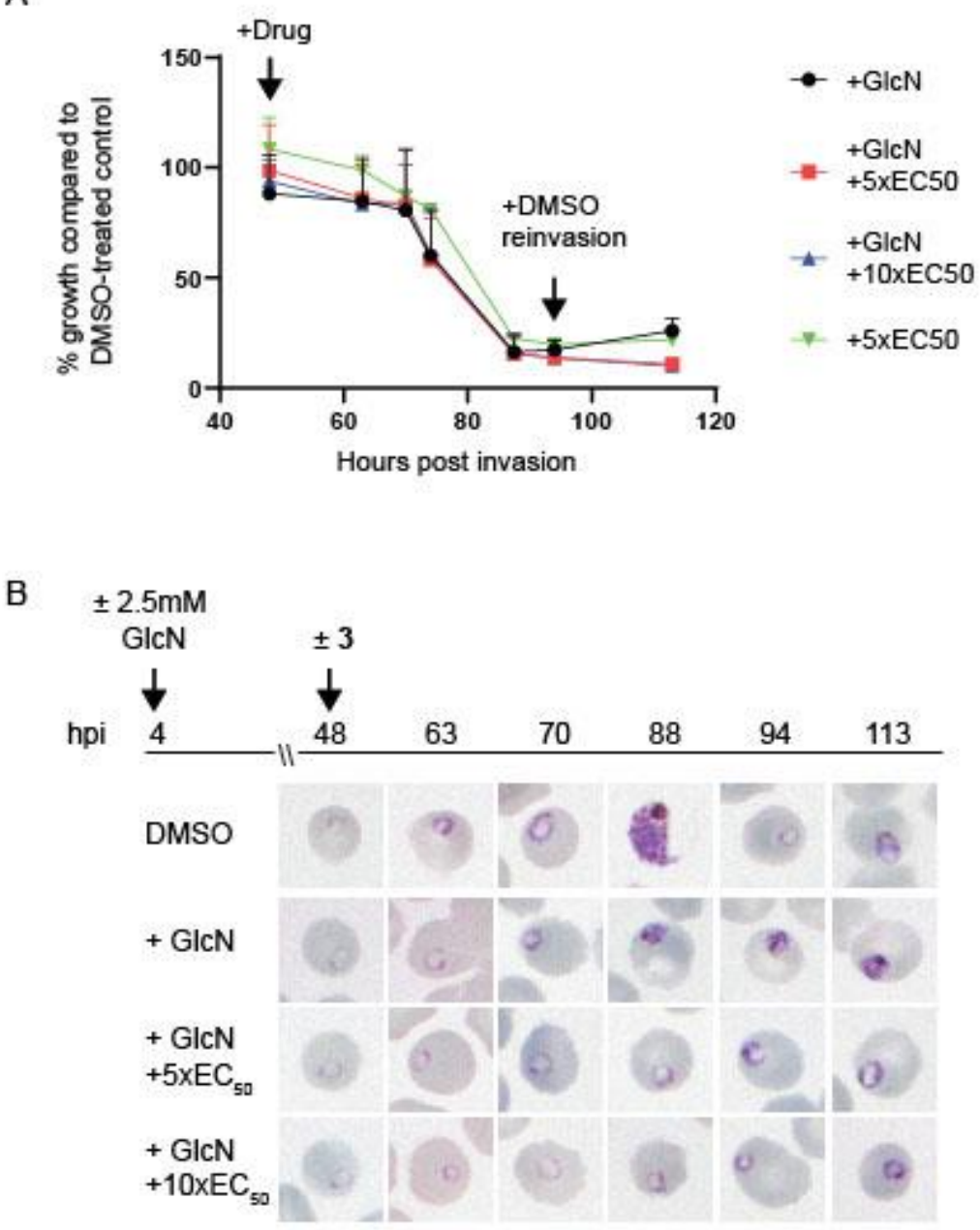

Figure 6. Effect of 3 treatment on parasites depleted of PfA-M17. (A) Percentage growth of PfAM17-HAglmS under different treatment conditions as indicated relative to the growth of DMSOtreated parasites. No significant difference was found between treatments. Plotted is the mean \pm standard deviation ( $\mathrm{n} \geq 2$ ). (B) Upper panel: overview of experiment. Heparin synchronized PfAM17-HAglmS parasites were treated with $2.5 \mathrm{mM}$ GlcN and allowed to invade into cycle 2 before being treated with 3 or DMSO. Lower panel: representative Giemsa-stained parasite smears of PfA-M17-HAglmS parasites treated with $\pm \mathrm{GlcN}$ and $\pm 5 x$ or $10 x \mathrm{EC}_{50}$ of compound 3 shows parasites developing at a slower rate than the DMSO-treated control but in a similar manner between the treatment groups, $\mathrm{n}=2$ biological replicates. 
bioRxiv preprint doi: https://doi.org/10.1101/2021.11.23.469631; this version posted November 23, 2021. The copyright holder for this preprint (which was not certified by peer review) is the author/funder. All rights reserved. No reuse allowed without permission.

A

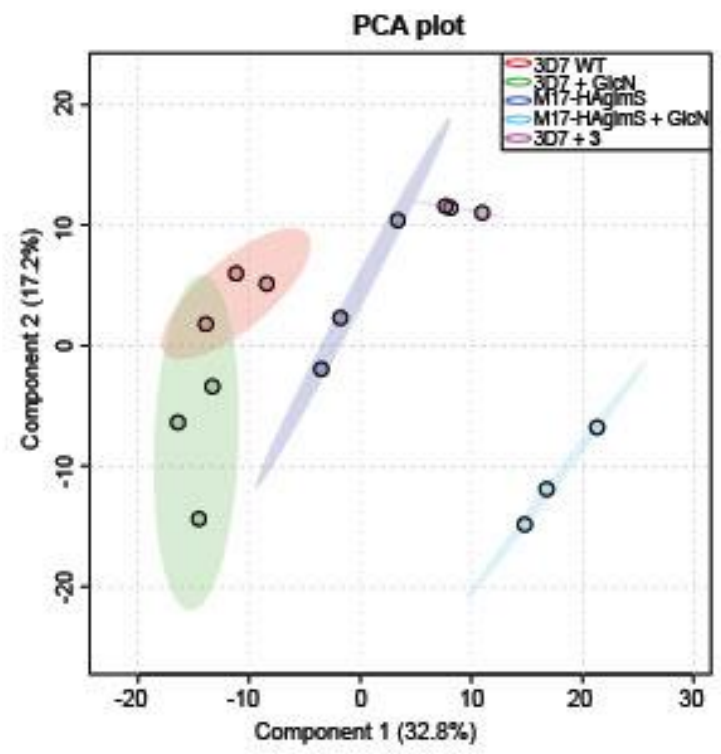

B

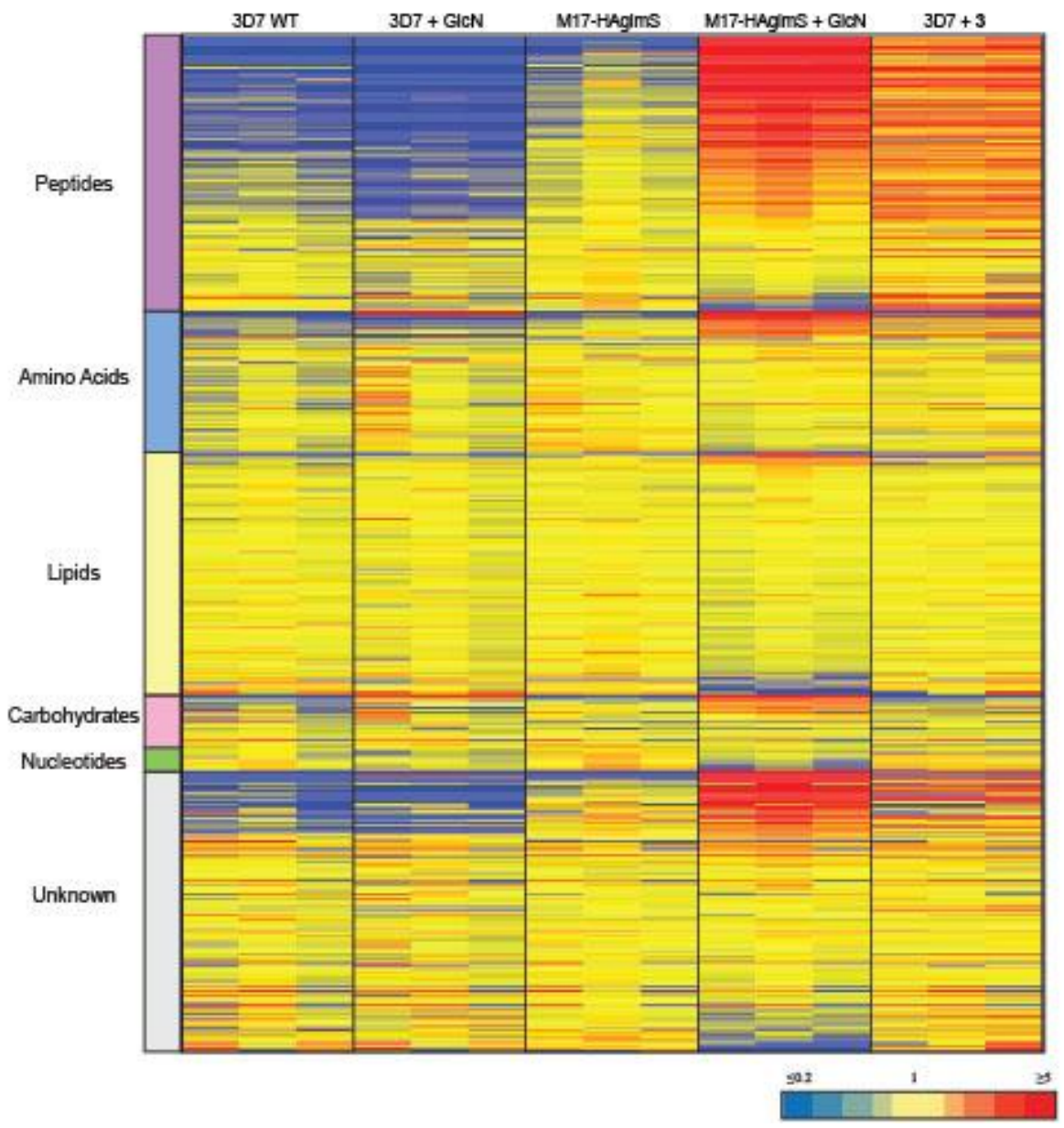


Figure 7. Untargeted metabolomics analysis of PfA-M17-HAglmS and Pf3D7 parasites treated with $\pm \mathrm{GlcN}$ and of Pf3D7 parasites treated with 3 from experiment 1. (A) Principal component analysis (PCA) of parasites (PfA-M17-HAglmS and Pf3D7) treated with +/- GlcN, 3 or DMSO control. Scores plot shows principal components one and two, data points indicate individual sample replicates within each condition and the shaded area denotes $95 \%$ confidence interval. (B) Heatmap analysis of normalized peak intensities of all putative metabolites for each condition. Data is shown from three technical replicates, red, blue and yellow indicates increase, decrease and no change respectively in the relative abundance of putative metabolites identified. 


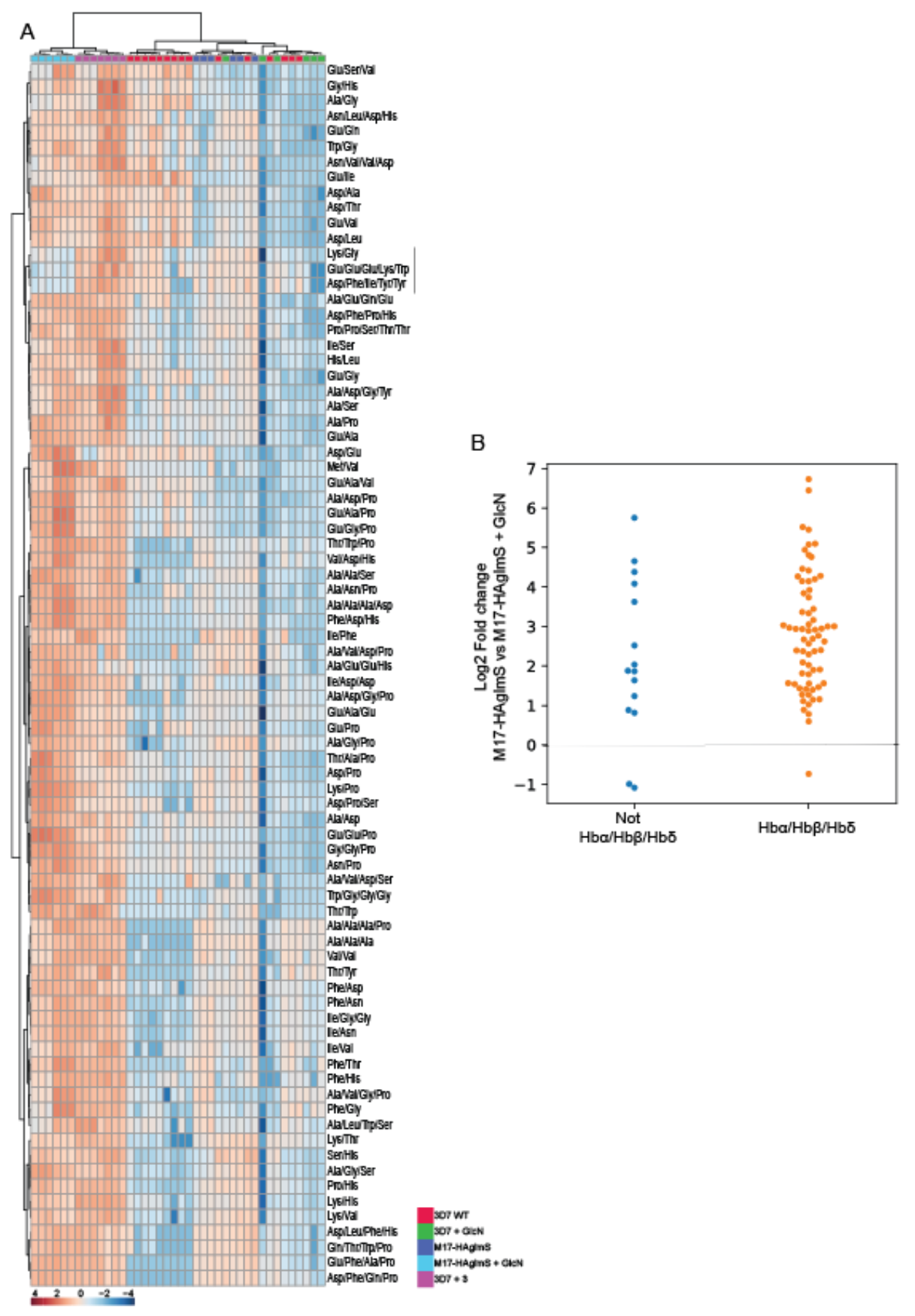

Figure 8. Targeted analysis of common significantly perturbed peptides ( $p$-value $<0.05)$ following addition of GlcN or treatment with 3 identified from experiment 1, 2 and 3. (A) Hierarchical clustering of the 80 significantly perturbed common peptides (fold change $>1.5$ and $p$-value $<0.05$ ) identified across the three independent experiments. Vertical clustering displays similarities between sample groups, while horizontal clusters reveal the relative abundances (median normalized) of the 80 peptides. The color scale bar represents $\log _{2}$ (mean-centered and divided by the standard deviation 
of each variable) intensity values. (B) Differential enrichment of the 80 common significantly perturbed peptides that could (orange dots) or could not (blue dots) be derived from hemoglobin $(\mathrm{Hb}) \alpha, \beta$, and $\delta$. 

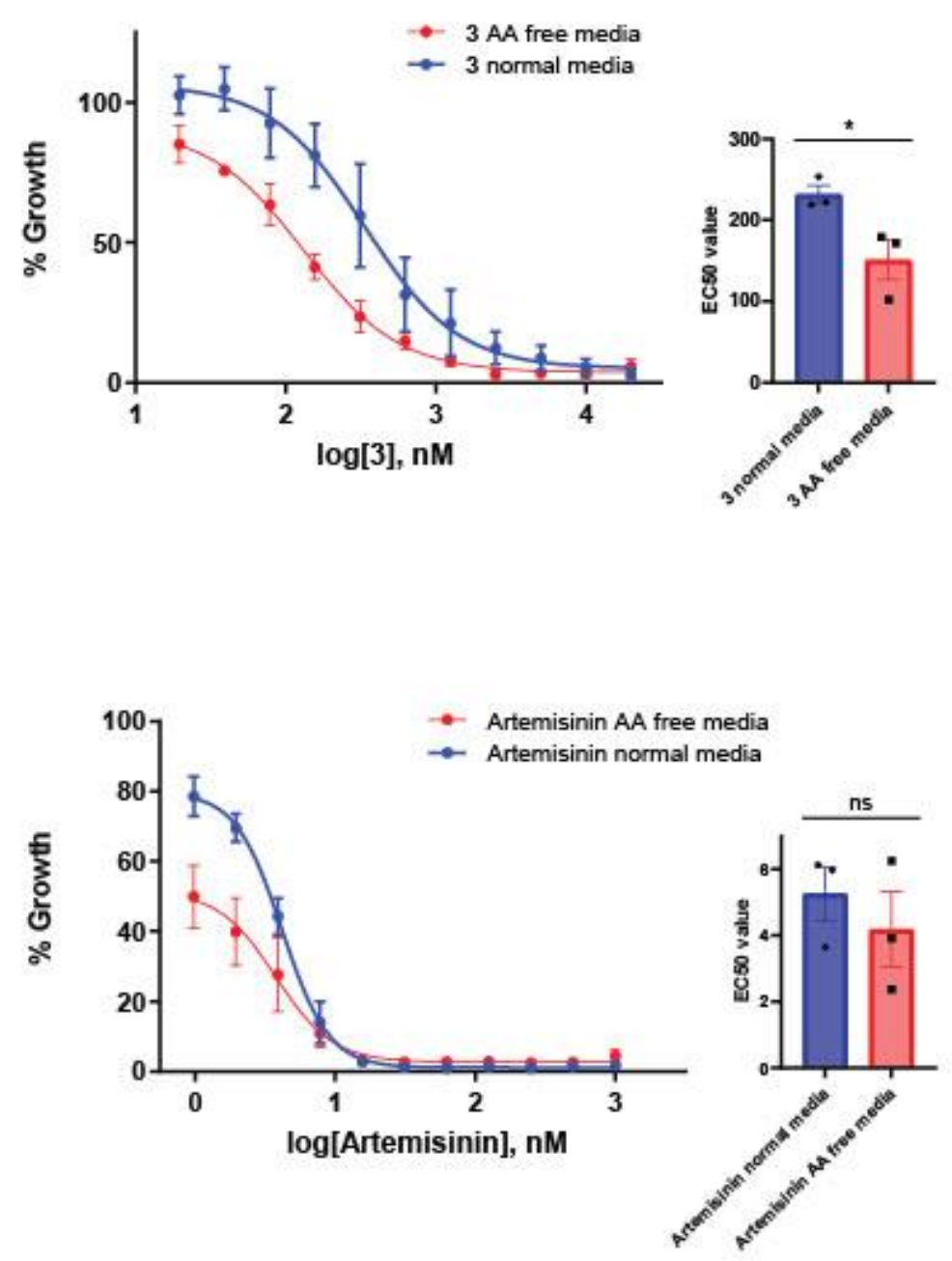

Figure 9. Removal of exogenous amino acids except for isoleucine sensitizes parasites to 3 . Killing action of $\mathbf{3}$ or Artemisinin in either normal medium containing all amino acids (blue) or amino acid (AA) free medium, containing isoleucine (red) was measured over $72 \mathrm{~h}$ and determined by SYBR Green I assay. The $\mathrm{EC}_{50}$ values were calculated from 3 biological replicates performed in triplicate and data plotted as the mean \pm standard error of the mean, with the inlay bar graphs showing the mean $\mathrm{EC}_{50}$ values of these replicates with statistical significance determined using an unpaired ttest, ${ }^{*} p \leq 0.05$. 